\title{
A Newtonian interpretation of configurational forces on dislocations and cracks
}

\author{
Roberto Ballarini ${ }^{1}$ and Gianni Royer-Carfagni ${ }^{2}$ \\ 1 Department of Civil and Environmental Engineering, University of Houston, TX, USA. rballarini@uh.edu, \\ 2 Department of Industrial Engineering, University of Parma, Italy. gianni.royer@unipt.it
}

\begin{abstract}
Configurational forces are fundamental concepts in the description of the motion of dislocations, cracks and other defects that introduce singularities within the solid state. They are defined by considering variations in energies associated with the movement of such defects, and are therefore different from the classical forces that enter the balance laws of classical Newtonian mechanics. Here, it is demonstrated how a configurational force can be viewed as the resultant of the (Newtonian) contact forces acting on the perturbed shape of an object of substance equivalent to the defect, and evaluated in the limit of the shape being restored to the primitive configuration. The expressions for the configurational forces onthe paradigmatic examples of cracks and dislocations are in agreement with those determined using classical variational arguments. This finding opens a new prospective in the use of configurational forces by permitting their physical and intuitive visualization.
\end{abstract}

Key words: Configurational forces; Eshelbian forces; Newtonian mechanics; dislocations; cracks; perturbative methods; J-integral.

\section{INTRODUCTION}

The states of stress and strain and their associated strain energy within an elastic body free of external actions is a subject that has been studied for more than one century. Such a condition may be produced, for example, by removing or adding a thin slice of material bounded by two planes and then rejoining the resulting material surfaces. The general theory of dislocations developed by Vito Volterra (Volterra, 1907), relying upon fundamental work of Ernesto Cesàro, permits the description of elastic states of this kind using a very limited number of variables including the elastic constants and the thickness and location of the geometric construct. In the atomistic physical theory of the solid state, the term "defect" more generally applies to different types of imperfections of the crystal lattice, which can develop and propagate as a consequence of the re-arrangement of the interatomic distances. The movement of imperfections is regulated by the neighboring environment, 
which may involve the presence of solute atoms in the crystalline lattice or the interaction with other defects, in such a way that the ensemble naturally evolves towards a minimum energy configuration.

Eshelby pioneered the use of the continuum theory of deformable bodies to describe, in mathematical terms, the potential movement of defects inside an elastic solid (Eshelby, 1951). Because the total energy of the system is a function of the parameters that are necessary to specify the nature and the position of the imperfection, he established the negative gradient of the total energy with respect to the position as the natural choice for the definition of the force driving the movement of the imperfection. However, the energetic state is calculated by solving the corresponding boundary value problem in elasticity theory for a fixed configuration of the imperfection, and therefore the resultant of the contact and body forces exerted on any arbitrary portion of the body is null. As Eshelby noted the aforementioned driving force "in a sense fictitious, is introduced to give a picturesque description of energy changes, and must not be confused with the ordinary surface and body forces acting on the material" (Eshelby, 1951). In order to emphasize such a distinction, actions of this kind are usually referred to as configurational forces.

Eshelby proposed an elegant way to calculate the configurational force on a defect that is associated with the variation of the elastic energy stored in the body (the energy release rate) as it changes its position. Specifically, he showed that this force can be written as the flux of a tensorial field $\mathbf{C}$, referred to as the energymomentum tensor, around a closed path enveloping the defect. The general expression for $\mathbf{C}$ is (Eshelby, 1975)

$$
\mathbf{C}=\Psi \mathbf{I}-\mathbf{F}^{T} \mathbf{S}
$$

where $\Psi$ is the elastic strain energy, $\mathbf{I}$ is the identity tensor, $\mathbf{S}$ is the first Piola-Kirchhoff stress tensor and $\mathbf{F}$ is the deformation gradient. An alternative systematic approach was proposed by Gurtin, who considered configurational forces as primitive objects, consistent with their own force balance, rather than as variational constructs (Gurtin, 1995). If one assumes the energy-momentum tensor $\mathbf{C}$ as a primitive object, for which the rate of work with the dual kinematic variable is defined, then the second law of thermodynamics furnishes for $\mathbf{C}$ the representation (1.1) as a consequence of invariance under re-parametrization of the referential control volume. It is very remarkable that in this approach dissipative phenomena, for which the variational derivation would be problematic, can also be naturally considered; for such problems the quantity $\Psi$ represents, more generally, the bulk free energy stored the body.

Configurational forces are basic concepts (Gurtin, 2008) and can be used to describe other physical phenomena such as evolving interfaces and material phases. The idea that the energy release rate is closely related to the configurational force on a defect was extended by Rice (Rice, 1968) to the case of a crack. Using the argument introduced by Eshelby, it is possible to relate the energy release rate to the flux of $\mathbf{C}$ on an arbitrary closed surface embracing the tip of the crack, the corresponding surface integral being referred to as the J-integral. Therefore, the energetic balance à la Griffith, assumed to govern crack extension, can be 
equivalently represented as the balance between the driving (configurational) force at the crack tip and the restoring force equal to the energy per unit area necessary to produce new surface via material separation. Within the context of the axiomatic framework of (Gurtin, 1995), this "configurational" force balance has been demonstrated for a very general class of materials (Gurtin \& Podio-Guidugli, 1996), also amenable of internal dissipation.

It is incontestable that configurational forces are less intuitive than the Newtonian forces used in classical mechanics to describe the equilibrium of a body, consistently with the balance laws for linear and angular momentum (Gurtin, 1995). As pointed out by Gurtin himself, "their characteristic feature is that they are related to the intrinsic coherency of a body's material structure, and perform work in the additional and removal of materials and in the evolution of structural defects". However, this definition is still not directly intuitive to the authors, because it is hard to imagine forces of distinct nature concurrently acting at each point of a body. It is equally hard to imagine how the source of a dislocation, or the tip of a crack, which are somehow immaterial, could be regarded as material entities over which such forces, albeit of a special character, should be applied.

Indeed, looking back at the history of mechanics, one discovers that Newtonian forces were superseded, for most purposes, by energy-based theories. D'Alambert spoke about them as "obscure and metaphysical beings, capable of nothing but spreading darkness over a science clear by itself" (Truesdell, 1966). In fact, Newtonian forces are nowadays commonly used by physicists and engineers only because they allow an intuitive representation based upon a simple geometric interpretation of the balance laws. Since Newton's laws may also be derived from Hamilton's principle, and Hamilton's principle may be derived from Newton's laws, from a theoretical point of view Newtonian mechanics and energy-based approaches to mechanics are mutually supporting, compatible perspectives on the phenomena of mechanical motions.

It appears useful for ontological, pedagogical and practical reasons to reconcile the energy-based calculation of the configurational forces acting on a defect with their possible derivation from the classical balance laws of Newtonian mechanics. This is what has been done in most branches of physical knowledge, including the whole field of electrostatic interactions (Coulomb forces, Maxwell stresses, etc.) that has been described in terms of equivalent "Newtonian forces" and "energy approaches", although the total energy of a point charge is found to be infinite, as infinite is the self energy of a point or line singularity in linear elasticity theory. A key point for the argument we are going to introduce is how to treat a "defect" as an "object of substance". For the case of dislocations, as done by Eshelby himself in his seminal work (Eshelby, 1951), one can establish a correspondence between the stress and strain state associated with a defect and that is produced by an equivalent "material inclusion", obtained by cutting and pasting new material in the reference matrix. For the case of a propagating sharp crack, it is convenient to consider it as the small scale bridging limit of a cohesive crack, and introduce a material region in which the cohesive forces are supposed to develop. Then, one has to observe that although in atomistic physical theories the constituent atoms are in equilibrium due to 
central forces exerted at a distance, in continuum theories the interaction between the various particles of the body is essentially due to contact forces. It is thus necessary to represent, in additional detail, the contact forces acting on the defect under consideration.

In recent years, Bigoni and co-workers have presented an interesting series of articles with experimental demonstrations discussing the equilibrium states of simple but paradigmatic elastic structures, composed of elastic beams with smooth bilateral constraints. If the constraint is allowed to move and modify the energetic state of the system, a force driving the configuration is generated that, in analogy with the energyderived forces acting on a dislocation, may be identified as "Eshelby-like" or "configurational" (Bigoni, et al., 2015). The value of such forces can be derived from a variational argument but, remarkably, it can also be found directly through an asymptotic approach based upon Newtonian force balance. This second approach, apparently first introduced in a forgotten article published in Russian (Balabukh, et al., 1970), consists in performing a perturbation of the constraints active on the structures. For the case of a beam sliding into a sleeve constraint (Bigoni, et al., 2015), it is sufficient to consider that the sleeve is imperfect, having a small gap $\Delta$, so that the perfect constraint is recovered for $\Delta \rightarrow 0$. If one considers the reactions of the imperfect constraint, it is simple to verify that they are not orthogonal to the undistorted configuration of the beam, and thus provide a non-null axial component. Remarkably, when $\Delta \rightarrow 0$ the force tends to become orthogonal but its magnitude tends to become infinite in such a way that the axial component approaches a non-zero limit that coincides with the configurational force obtained with energy-based arguments. The result has been corroborated through numerous illustrative experiments.

We have drawn inspiration from the aforementioned work, because an argument of this kind provides a physical interpretation of the configurational forces acting on moving elastic structures from the point of view of classical Newtonian mechanics. The three different-in-type derivations ("variational", "Newtonian" and "experimental") of the Eshelby-like forces have been developed for a few other structural problems, such as the instability of a penetrating blade (Bigoni, et al., 2014) and the injection of an elastic rod (Bosi, et al., 2015). Interesting applications range from the innovative concept-design of an elastica arm-scale (Bosi, et al., 2014), to the possibility of locomotion using the expulsive (configurational) force exerted on a twisted elastic beam inserted in a frictionless fitting socket (Bigoni, et al., 2014) and, more recently, to self-encapsulation (or "dripping") of an elastic rod (Bosi, et al., 2015).

The aim of this article is to present a Newtonian view of the configurational forces acting on dislocations and cracks. The proposed method is consistent with the procedure used for the elastic structures in (Bigoni, et al., 2015), since it essentially consists of calculating the (Newtonian) forces on a slightly perturbed configuration of the defect modulo a proper definition of the nature of the contact forces. It is shown that while recovering the primitive configuration, the resultant of such forces tends to a definite limit, coinciding with the configurational forces exerted on it, calculated from energetic considerations. In order to render the argument clearer, first the aforementioned conclusion is proved on simplified models, including a 
blade penetrating an elastically fitting sleeve (Section 2.1) as a representation of an edge dislocation, and a double beam layout (Section 3.1) that is representative of a crack. The proposed perturbation approach allows a direct and arguably more physical interpretation of the configurational forces acting on an edge dislocation (Section 2.2) as it interacts with other defects (Peach-Koheler formula) or a free surface. For the case of cracks (Section 3.2), the perturbation consists in assuming that the tip of the crack is bridged by cohesive forces $\grave{a} l a$ Barenblatt (Barenblatt, 1962), and in calculating the (Newtonian) forces acting on the object of substance embracing the ligaments producing the cohesion. In fact, such ligaments are identified with a material portion in a neighborhood of the crack tip, usually referred to as the process zone. In the small-scale bridging limit one can recover the configurational force calculated using the J-integral approach. Such force acts on the crack tip, as this represents the image of the process zone in the aforementioned limit. The approach is more rigorously formalized Section 4, by referring to the general case of a Somigliana dislocation (Section 4.1), and by proposing an interpretation of the nature of the contact forces between the elastic matrix and the equivalent inclusion, within a boundary layer approximation (Section 4.2).

In the following, we shall let $\Omega \subset \mathbb{R}^{d}$, represent the undistorted reference configuration of an elastic body of dimension $d$. Then $\mathbf{y}(\mathbf{x}): \Omega \rightarrow \Omega^{*} \subset \mathbb{R}^{d}$ will denote the deformation of $\Omega$, i.e., the position of a particle initially at $\mathbf{x}$. Furthermore, we will indicate with $\mathbf{u}(\mathbf{x})=\mathbf{y}(\mathbf{x})-\mathbf{x}$ the displacement field and with $\mathbf{F}=\nabla \mathbf{y}=$ $\mathbf{I}+\nabla \mathbf{u}$ the deformation gradient. The following considerations rely on the energy-momentum tensor $\mathbf{C}$, defined in (1.1). Suppose that a defect (e.g., an edge dislocation), is present and that the total energy of the body depends upon the position of a reference point (e.g., the tip of the dislocation). Then, it is possible to show (Eshelby, 1975) that when body forces are negligible, the strain energy release rate associated with the movement of the defect in the direction indicated by the vector $\mathbf{r}$, which coincides with the component $R_{r}$ of the configurational force $\mathbf{R}$, reads

$$
R_{r}=\mathbf{r} \cdot \mathbf{R}=\mathbf{r} \cdot \int_{\Gamma}\left(\Psi \mathbf{I}-\mathbf{F}^{T} \mathbf{S}\right) \mathbf{n} d s=\int_{\Gamma}(\Psi \mathbf{r} \cdot \mathbf{n}-\mathbf{F r} \cdot \mathbf{S n}) d s
$$

where $\Gamma$ is a closed surface enveloping the defect itself. Such a relationship can be verified also under very general hypotheses, assuming proper balance laws for the configurational forces through the energymomentum tensor $\mathbf{C}$ and an invariance argument (Gurtin, 2008).

Recalling that the flux of $\mathbf{S}$ is null when body forces are not present, then (1.2) can be equivalently be written in the form

$$
R_{r}=\mathbf{r} \cdot \mathbf{R}=\mathbf{r} \cdot \int_{\Gamma}\left(\Psi \mathbf{I}-\nabla \mathbf{u}^{T} \mathbf{S}\right) \mathbf{n} d s=\int_{\Gamma}(\Psi \mathbf{r} \cdot \mathbf{n}-\nabla \mathbf{u r} \cdot \mathbf{S n}) d s
$$


In the simplest case of linear elastic materials, for which it is supposed that $|\nabla \mathbf{u}|<<1$, the first Piola-Kirchhoff stress tensor coincides with the Cauchy stress tensor up to quantities infinitesimal of the first order.

We will introduce an orthogonal reference system $\left(x_{1}, x_{2}, x_{3}\right)$ with corresponding unit vectors $\left(\mathbf{e}_{1}, \mathbf{e}_{2}, \mathbf{e}_{3}\right)$, and we will indicate the components of deformation and displacement by setting

$$
\mathbf{y}(\mathbf{x})=y_{i}\left(x_{1}, x_{2}, x_{3}\right) \mathbf{e}_{i}, \quad \mathbf{u}(\mathbf{x})=u_{i}\left(x_{1}, x_{2}, x_{3}\right) \mathbf{e}_{i},
$$

with an obvious specialization for the case of plane problems. Partial differentiation with respect to the representative variable will be indicated by comma, so that, in components

$$
(\nabla \mathbf{u})_{i j}=\frac{\partial u_{i}}{\partial x_{j}}=u_{i},{ }_{j} .
$$

In all the expressions, repeated indices will indicate summation.

\section{CONFIGURATIONAL FORCES ON EDGE DISLOCATIONS}

In order to introduce our interpretation of configurational forces acting on edge dislocation from the point of view of simple balance of the standard forces, it is useful to study first an elementary model. This contains all the basic features of the underlying rationale, but it is much more intuitive than the corresponding problem in the mathematical theory of elasticity, which will be considered later.

\subsection{The elementary model of a dislocation}

Consider an elementary model problem in which $\Omega$ is the special orthotropic two-dimensional body (Figure 1) composed of a set of long bars, separated by frictionless rollers, that can deform only along the $x_{2}$ axis (zero Poisson's ratio). Consequently the stress in the body is uniaxial. The body occupies the region $\left\{0 \leq x_{1} \leq l,-h / 2 \leq x_{2} \leq h / 2\right\}$ and the bars are clamped on the sides $x_{2}= \pm h / 2$, whereas only the $x_{1}$-component of displacement is constrained on the side $x_{1}=l$.

Now suppose that the bars are cut in the middle section $x_{2}=0$ and that a rigid and frictionless lamella, of thickness $b$, is inserted inside the body for the distance $\xi$. A sketch of the resulting configuration is represented in Figure 1. In this simplified view, the lamella penetrating inside the body is representative of a dislocation with Burger's vector of norm $b$. 


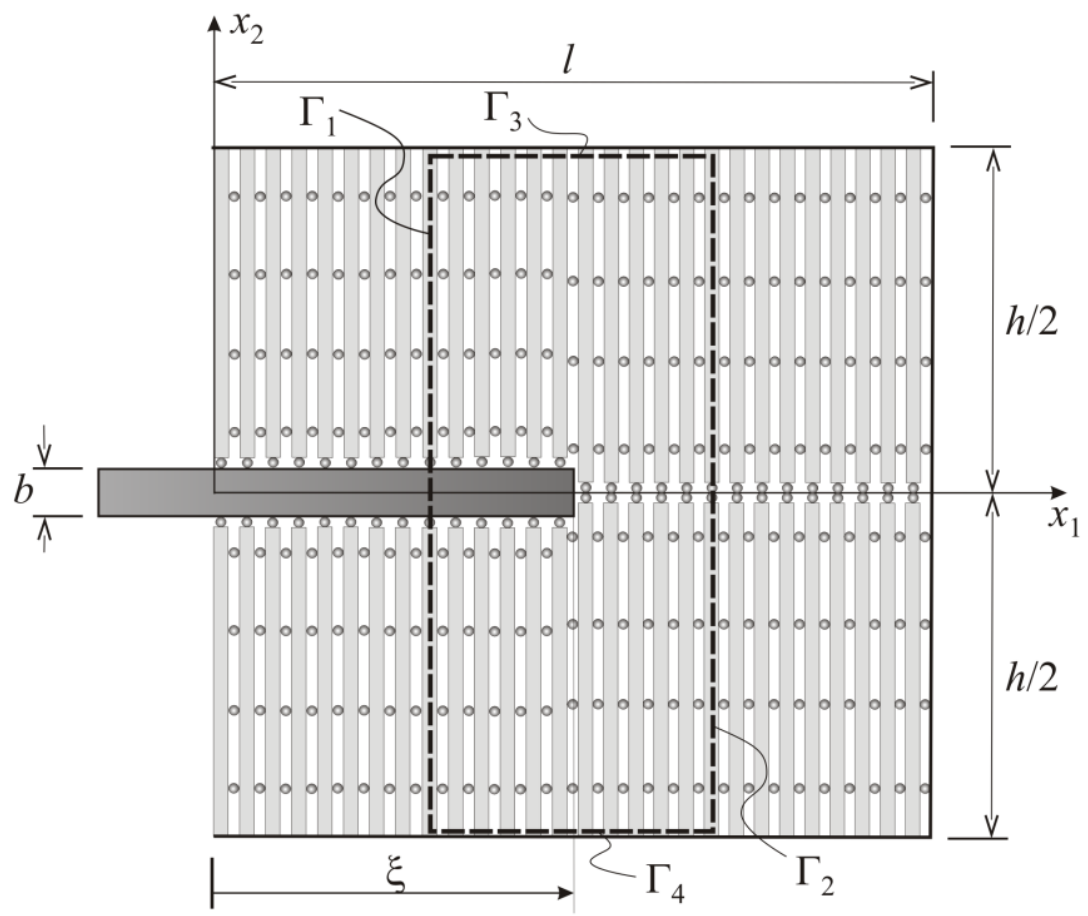

Figure 1. Schematic representation of the elementary model.

Although, for the sake of illustration, the bars have been drawn with a non-zero width, we will make the assumption that such width is much smaller than $b$. This allows a smeared view of the system and the use of a continuum description. Let $E$ represent the elastic modulus of the bars, and let $t$ denote their thickness, supposed constant, in the $x_{3}$ direction (at right angle to the plane of representation). The elastic strain energy of the body, after the insertion of the lamella, reads

$$
\Pi=\frac{1}{2} E \xi t 2 \frac{h}{2}\left(\frac{b}{h}\right)^{2}=\frac{1}{2} E \xi t \frac{b^{2}}{h} .
$$

The system of Figure 1 is in equilibrium, since the resultant of the contact forces applied to the lamella is zero whatever the depth of penetration $\xi$. However, a configurational force in the $x_{1}$ direction does exist, whose form can be obtained, from differentiation of the strain energy (2.1), as

$$
\hat{R}_{x 1}=-\frac{\partial \Pi}{\partial \xi}=-\frac{1}{2} E t \frac{b^{2}}{h} .
$$

Such an expression can be obtained from a direct application of the Eshelby energy-momentum tensor in the form (1.2), or equivalently (1.3). Consider the rectangular path $\Gamma=\Gamma_{1} \cup \Gamma_{2} \cup \Gamma_{3} \cup \Gamma_{4}$ shown in Figure 1 , where $\Gamma_{3}$ and $\Gamma_{4}$ are taken in a neighborhood of the borders $x_{2}=h / 2$ and $x_{2}=-h / 2$, respectively. Denoting 
with $\mathbf{n}$ the outer unit normal to the domain enclosed by $\Gamma$, one obtains by setting $\mathbf{r}=\mathbf{e}_{1}$

$$
R_{x 1}=\mathbf{e}_{1} \cdot \int_{\Gamma}\left(\Psi \mathbf{n}-\mathbf{F}^{T} \mathbf{S n}\right) t d s=\int_{\Gamma}\left(\Psi \mathbf{n} \cdot \mathbf{e}_{1}-\mathbf{S n} \cdot \mathbf{F} \mathbf{e}_{1}\right) t d s .
$$

But on $\Gamma_{3}$ and $\Gamma_{4}$ one has $\mathbf{n} \cdot \mathbf{e}_{1}=0$, while $\mathbf{n} \cdot \mathbf{e}_{1}=-1$ and $\mathbf{n} \cdot \mathbf{e}_{1}=+1$ on $\Gamma_{1}$ and $\Gamma_{2}$, respectively. On the other hand, $\Psi=0$ on $\Gamma_{2}$ because, here, the bars are not strained. Moreover, $\mathbf{S n}=\mathbf{0}$ on $\Gamma_{1}$ and $\Gamma_{2}$, whereas $\mathbf{S n}=E b / h$ $\mathbf{n}$ on $\Gamma_{3}$ and $\Gamma_{4}$. Therefore $\mathbf{S n} \cdot \mathbf{F} \mathbf{e}_{1}=E b / h y_{2,1} \mathbf{n} \cdot \mathbf{e}_{2}$ on $\Gamma_{3}$ and $\Gamma_{4}$, but such expression is null since $y_{2}$ is constant on the constrained borders $\left(y_{2,1}=0\right)$. Therefore, the only surviving term in (2.3) is the integral of $\Psi$ on $\Gamma_{1}$, which reads

$$
R_{x 1}=-\int_{\Gamma_{1}} \Psi t d s=-\frac{1}{2} E t\left(\frac{b}{h}\right)^{2} 2 \frac{h}{2}=-\frac{1}{2} E t \frac{b^{2}}{h}
$$

Since $R_{x 1}=\hat{R}_{x 1}$, this represents an alternative derivation of the expression (2.2).

There is obviously an apparent paradox in the occurrence of the force $R_{x 1}$, since the resultant of the contact forces on the lamella, represented in Figure 1, is clearly zero. On the other hand, the Eshelby approach clearly indicates that the lamella would tend to be expelled from the body. A somehow similar paradox, for the penetration of a lamella inside a sliding sleeve, has been illustrated in (Bigoni, et al., 2015).

In order to physically visualize the configurational force by using Newtonian forces, consider a perturbation of the tip of the lamella in the region $\xi-\alpha \leq x_{1} \leq \xi$, as represented in Figure 2a.

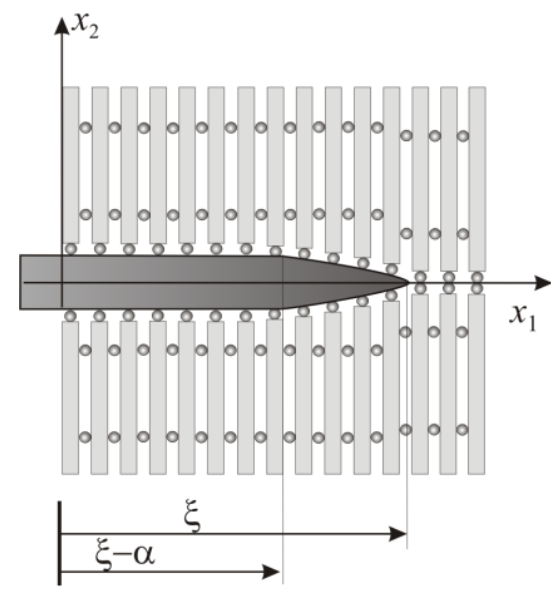

(a)

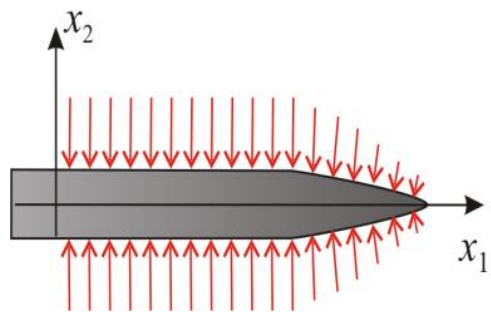

(b)

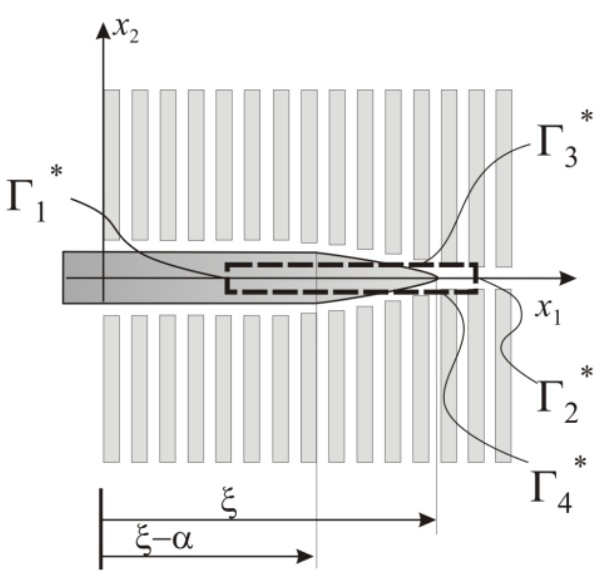

(c)

Figure 2. (a) Perturbation of the tip of the lamella. (b) Forces acting at the perturbed tip. (c) Pillow path for the integration of the energy-momentum tensor in the reference configuration.

Suppose for convenience, but without loosing generality, that the perturbation is symmetric with respect 
to the $x_{1}$ axis. The perturbation is completely described by the deformation component

$$
y_{2}\left(x_{1}, 0^{+}\right)=-y_{2}\left(x_{1}, 0^{-}\right), \quad \xi-\alpha \leq x_{1} \leq \xi .
$$

The forces in the bars touching the perturbed tip are not constant because the corresponding axial strain is varying (Figure $2 b$ ). Under the hypothesis that the contact between the bars and the lamella is frictionless, one has that the contact forces are normal to the perturbed tip-surface. As represented in Figure 3, such forces can be decomposed into vertical components which are equilibrated by the axial stress in the constituent bars, and into horizontal components which tend to pull the lamella leftwards. In an elementary bar fiber of width $d x_{1}$ the compressive axial force, coinciding with the vertical component of the aforementioned contact force, is of the form

$$
d f=E t \frac{y_{2}\left(x_{1}, 0^{+}\right)-y_{2}\left(x_{1}, 0^{-}\right)}{h} d x_{1}=2 E t \frac{y_{2}\left(x_{1}, 0^{+}\right)}{h} d x_{1}, \quad \xi-\alpha \leq x_{1} \leq \xi .
$$

The resultant of the horizontal components represents an action that tends to expel the lamella from the body, and needs to be equilibrated by external actions.

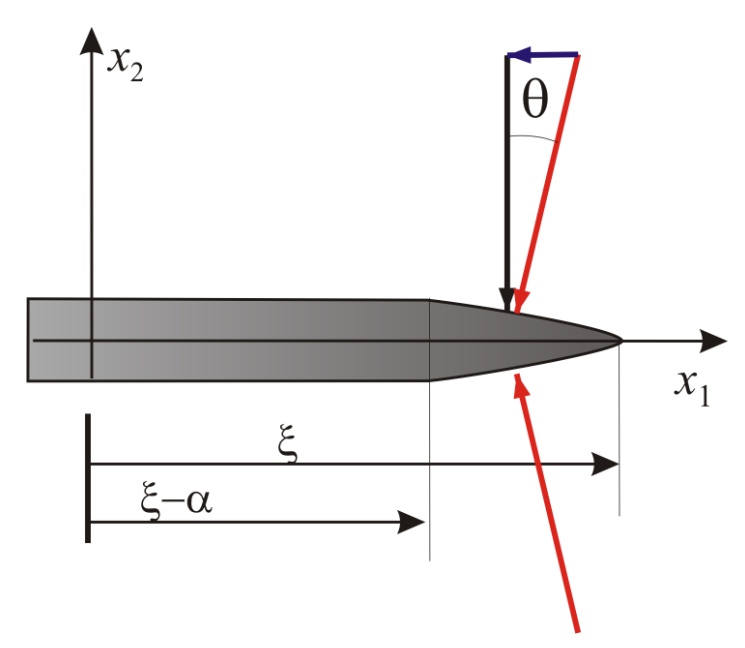

Figure 3. Decomposition of the contact force between the bars and the perturbed tip of the lamella into normal and horizontal components.

Observing from Figure 3 that

$$
\tan \theta=-\frac{\partial}{\partial x_{1}} y_{2}\left(x_{1}, 0^{+}\right)=\frac{\partial}{\partial x_{1}} y_{2}\left(x_{1}, 0^{-}\right), \quad \xi-\alpha \leq x_{1} \leq \xi,
$$

the resultant of the horizontal components reads 


$$
\bar{R}_{x 1}=-2 \int_{\xi-\alpha}^{\xi} 2 E t \frac{y_{2}\left(x_{1}, 0^{+}\right)}{h} \tan \theta d x_{1}=\frac{2 E t}{h} \int_{\xi-\alpha}^{\xi} \frac{\partial}{\partial x_{1}}\left(y_{2}\left(x_{1}, 0^{+}\right)\right)^{2} d x_{1}=\frac{2 E t}{h}\left[\left(y\left(x_{1}, 0^{+}\right)\right)^{2}\right]_{\xi-\alpha}^{\xi}=-\frac{1}{2} E t \frac{b^{2}}{h} .
$$

Clearly, this values is identical to that obtained in (2.2) and (2.4).

It should be remarked that this finding is independent of the particular form of perturbation used. In other words, a whatever "small" variation of the profile of the tip of the lamella would result in a force tending to expel the lamella itself from the elastic body. A key hypothesis for this result is that the contact between the elastic matrix and the lamella is frictionless.

It is worth observing that the same result of (2.8) could be obtained by a direct application of the Eshelby energy-momentum tensor to the perturbed lamella-tip. In the reference undistorted configuration of the body, consider the pillow path $\Gamma^{*}=\Gamma_{1}{ }^{*} \cup \Gamma_{2}{ }^{*} \cup \Gamma_{3}{ }^{*} \cup \Gamma_{4}{ }^{*}$ represented in Figure $2 \mathrm{c}$.

The corresponding configurational force, $R_{x 1}^{*}$, takes the same form of (2.3) with $\Gamma$ substituted by $\Gamma^{*}$. Again, $\mathbf{n} \cdot \mathbf{e}_{1}=0$ on $\Gamma_{3}{ }^{*}$ and $\Gamma_{4}{ }^{*}$, while the length of $\Gamma_{1}{ }^{*}$ and $\Gamma_{2}{ }^{*}$ can be made infinitesimally small. Consequently, the term in the integral containing $\Psi$ vanishes. The only non-zero contribution to the second term comes from integration on $\Gamma_{3}{ }^{*}$ and $\Gamma_{4}{ }^{*}$. Observing that $y_{2}\left(x_{1}, 0^{+}\right)$and $y_{2}\left(x_{1}, 0^{-}\right)$are constant for $x_{1} \notin[\xi-\alpha, \xi]$, one obtains

$$
\begin{aligned}
& R_{x 1}^{*}=-\int_{\Gamma^{*}} \mathbf{S n} \cdot \mathbf{F} \mathbf{e}_{1} t d s=\frac{2 E t}{h}\left[\int_{\Gamma_{3}^{*}} y_{2}\left(x_{1}, 0^{+}\right) \frac{\partial}{\partial x_{1}}\left(y_{2}\left(x_{1}, 0^{+}\right)\right) d x_{1}+\int_{\Gamma_{4}^{*}} y_{2}\left(x_{1}, 0^{-}\right) \frac{\partial}{\partial x_{1}}\left(y_{2}\left(x_{1}, 0^{-}\right)\right) d x_{1}\right] \\
& =\frac{E t}{h} \int_{\xi-\alpha}^{\xi} \frac{\partial}{\partial x_{1}}\left[\left(y_{2}\left(x_{1}, 0^{+}\right)\right)^{2}+\left(y_{2}\left(x_{1}, 0^{-}\right)\right)^{2}\right] d x_{1}=\frac{E t}{h}\left(-\frac{b^{2}}{4}-\frac{b^{2}}{4}\right)=-\frac{1}{2} E t \frac{b^{2}}{h} .
\end{aligned}
$$

This result is identical to (2.8) and, again, it is independent of the perturbation of the tip of the lamella.

One could select a borderline perturbation coinciding with the Heaviside function ${ }^{1}$

$$
y_{2}\left(x_{1}, 0^{+}\right)=-y_{2}\left(x_{1}, 0^{-}\right)=\frac{b}{2} H\left(\xi-x_{1}\right) .
$$

Then (2.9) becomes

$$
R_{x 1}^{*}=-\int_{\Gamma^{*}} \mathbf{S n} \cdot \mathbf{F e}_{1} t d s=\frac{E t b^{2}}{h} \int_{\xi^{-}}^{\xi^{+}}\left[H\left(\xi-x_{1}\right) \frac{\partial}{\partial x_{1}}\left(H\left(\xi-x_{1}\right)\right)\right] d x_{1}=\frac{E t b^{2}}{2 h}\left[\left(H\left(\xi-x_{1}\right)\right)^{2}\right]_{\xi^{-}}^{\xi^{+}}=-\frac{1}{2} E t \frac{b^{2}}{h} .
$$

This limit result has been obtained by using a step function, which can be considered as the limit of a sequence of smooth perturbations.

\footnotetext{
${ }^{1}$ The Heaviside function $H(t)$ is equal to 1 if $t>0$ and to -1 if $t<0$. The generalized derivative, in the sense of distributions, of the Heaviside function is the Dirac's delta function $\delta(t)$.
} 
In conclusion, we have directly recovered the expression of the configurational forces acting on the lamella in four different ways: $i$ ) from direct energy derivation (expression (2.2) for $\hat{R}_{x 1}$ ); $i i$ ) with an application of Eshelby's energy-momentum tensor (expression (2.4) for $\hat{R}_{x 1}$ ); iii) from standard equilibrium, as the unbalanced resultant of the contact forces on the perturbed lamella (expression (2.8) for $\bar{R}_{x 1}$ ); iv) by the contour integral of Eshelby's energy-momentum tensor upon the perturbed tip of the lamella (expression (2.11) for $\bar{R}_{x 1}^{*}$ ). The derivations iii) and iv) also hold in the limit of a rectangular, unperturbed lamella, i.e., when the original configuration is restored.

\subsection{Forces on edge dislocations}

The same perturbation approach presented in Section 2.1 is now applied to various types of dislocations in an elastic continuum. To render them amenable to closed form solutions the dislocations are considered to lie within the linear elasticity theory framework so that, in (1.2) or (1.3), $\mathbf{S}$ can be confused with the Cauchy stress tensor, and the deformed configuration coincides with the undistorted reference state.

\subsubsection{Edge dislocation in an infinite elastic solid}

As schematically represented in Figure 4a, consider the plane problem of an edge dislocation with Burger's vector $\mathbf{b}=b_{2} \mathbf{e}_{2}$, laying in an infinite, homogeneous and isotropic, linear elastic medium. In polar coordinates, the state of stress (Dundurs, 1969) is of the form

$$
\sigma_{r r}=\sigma_{\theta \theta}=\frac{2 G b_{2}}{\pi(1+\kappa)} \frac{\cos \theta}{r}, \tau_{r \theta}=\frac{2 G b_{2}}{\pi(1+\kappa)} \frac{\sin \theta}{r},
$$

where $G$ is the elastic shear modulus and, denoting with $v$ the Poisson's ratio, $\kappa=3-4 v$ for plane strain $(\kappa=$ $(3-v) /(1+v)$ for generalized plane stress) conditions. 


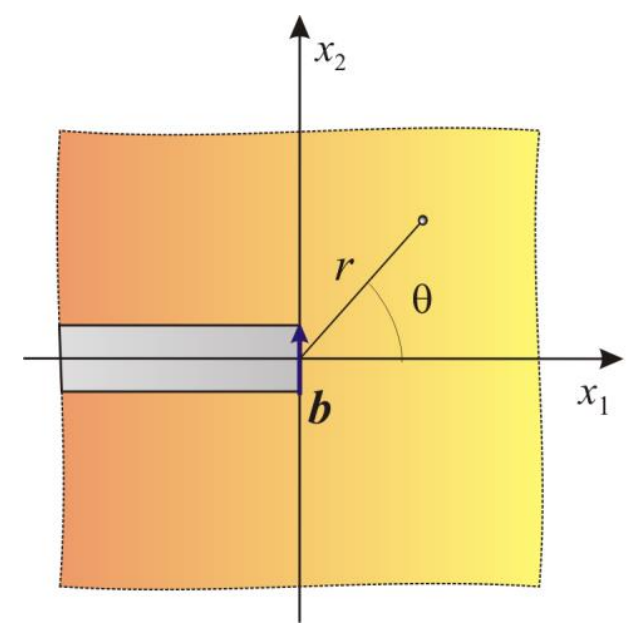

(a)

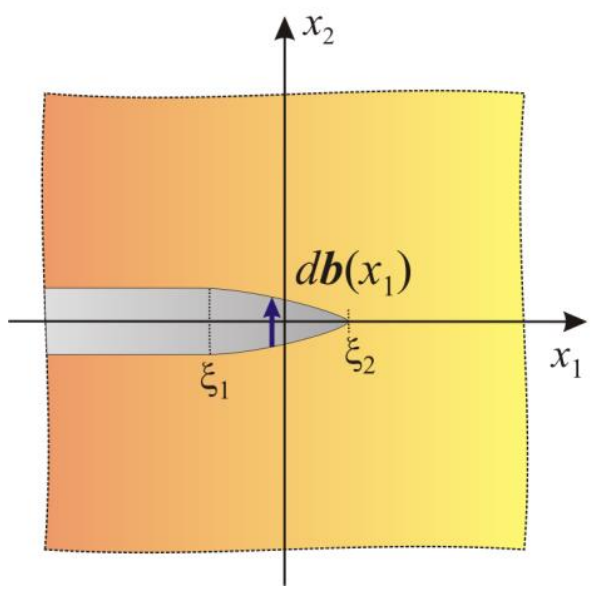

(b)

Figure 4. (a) The edge dislocation in an infinite medium. (b) The perturbed dislocation.

From the analogy of the penetration of a lamella, we perform a perturbation of the dislocation tip by a considering, instead of a sharp discontinuity, a distributed dislocation along the $x_{1}$ axis, with dislocation density $\rho\left(x_{1}\right)$, such that the Burger's vector associated with the infinitesimal dislocation applied on the element $d x_{1}$ reads

$$
d \mathbf{b}=\rho\left(x_{1}\right) d x_{1} \mathbf{e}_{2}, \quad \xi_{1} \leq x_{1} \leq \xi_{2},
$$

where the interval $\left(\xi_{1}, \xi_{2}\right)$ lays in a small neighborhood of $x_{1}=0$. A condition to be satisfied is that

$$
\int_{\xi_{1}}^{\xi_{2}} d \mathbf{b}=\mathbf{e}_{2} \int_{\xi_{1}}^{\xi_{2}} \rho\left(x_{1}\right) d x_{1}=b_{2} \mathbf{e}_{2} \text {. }
$$

A schematic representation of the perturbed dislocation tip is drawn in Figure $4 \mathrm{~b}$. The corresponding $x_{2}$ component of stress on the line $x_{2}=0$ reads

$$
\sigma_{22}\left(x_{1}, 0\right)=\int_{\xi_{1}}^{\xi_{2}} \frac{2 G}{\pi(1+\kappa)} \frac{\rho(\xi)}{x_{1}-\xi} d \xi .
$$

On the other hand, the deformation jump on the line $x_{1}=0$ due to the distributed dislocation takes the form

$$
y_{2}\left(x_{1}, 0^{+}\right)-y_{2}\left(x_{1}, 0\right)=\int_{\xi_{1}}^{\xi_{2}} \rho(\xi) H\left(\xi-x_{1}\right) d \xi,
$$

where the Heaviside function has been defined in footnote 1. Then 


$$
\frac{\partial}{\partial x_{1}}\left(y_{2}\left(x_{1}, 0^{+}\right)-y_{2}\left(x_{1}, 0\right)\right)=-\int_{\xi_{1}}^{\xi_{2}} \rho(\xi) \delta\left(\xi-x_{1}\right) d \xi=-\rho\left(x_{1}\right),
$$

where $\delta(\cdot)$ represents the Dirac's delta function. Reasoning as in (2.7) and (2.8), in particular assuming a "frictionless contact", the force exerted on the perturbed dislocation reads

$$
\begin{aligned}
\bar{R}_{x 1} & =-\int_{\xi_{1}}^{\xi_{2}} \sigma_{22}\left(x_{1}, 0\right) \frac{\partial}{\partial x_{1}}\left(y_{2}\left(x_{1}, 0^{+}\right)-y_{2}\left(x_{1}, 0^{-}\right)\right) d x_{1}=\int_{\xi_{1}}^{\xi_{2}}\left(\int_{\xi_{1}}^{\xi_{2}} \frac{2 G}{\pi(1+\kappa)} \frac{\rho(\xi)}{x_{1}-\xi} d \xi\right) \rho\left(x_{1}\right) d x_{1} . \\
& =\int_{\xi_{1}}^{\xi_{2}} \int_{\xi_{1}}^{\xi_{2}} \frac{2 G}{\pi(1+\kappa)} \frac{\rho(\xi) \rho\left(x_{1}\right)}{x_{1}-\xi} d \xi d x_{1} .
\end{aligned}
$$

This expression is clearly null if the integral is interpreted as being of the Cauchy's principal value type, because the integration domain in the $\left(x_{1}, \xi\right)$ is symmetric with the respect to the line $x_{1}=\xi$, whereas the integrand is antisymmetric with respect to the same line.

Analogous to (2.9), one can obtain the same result by a direct application of Elshelby's energy momentum tensor, by using the pillow path $\Gamma^{*}=\Gamma_{1}{ }^{*} \cup \Gamma_{2}{ }^{*} \cup \Gamma_{3}{ }^{*} \cup \Gamma_{4}{ }^{*}$ analogous to that represented in Figure 2c, which embraces the interval $\xi_{1} \leq x_{1} \leq \xi_{2}$ on the line $x_{2}=0$. Clearly $\mathbf{n} \cdot \mathbf{e}_{1}=0$ on $\Gamma_{3}{ }^{*}$ and $\Gamma_{4}{ }^{*}$, while the length of $\Gamma_{1}{ }^{*}$ and $\Gamma_{2}{ }^{*}$ can be made infinitesimally small so that the term associated with the strain energy $\Psi$ is null, because a perturbation can be chosen such that the stress field results to be uniformly continuous. In conclusion, one obtains

$$
R_{x 1}^{*}=-\int_{\Gamma^{*}} \mathbf{S n} \cdot \mathbf{F} \mathbf{e}_{1} t d s=-\int_{\xi_{1}}^{\xi_{2}} \sigma_{22}\left(x_{1}, 0\right) \frac{\partial}{\partial x_{1}}\left[y_{2}\left(x_{1}, 0^{+}\right)-y_{2}\left(x_{1}, 0^{-}\right)\right] d x_{1},
$$

which coincides with (2.18).

It is emphasized that the resulting force is independent of the particular perturbation that is assumed for the dislocation tip, since the final expression is independent of the form of $\rho(\xi)$ as well as the choice of the points $\xi_{1}$ and $\xi_{2}$. Therefore, the same conclusion holds in the limit case in which the initial unperturbed configuration is restored. The fact that the configurational force, so calculated, vanishes for on an edge dislocation in an infinite medium is in agreement with the classical result obtained with energy-based arguments (Dundurs, 1969).

2.2.2 Edge dislocation in an infinite solid under an applied stress field (Peach-Koheler formula) 
Consider again the edge dislocation in an infinite elastic medium of Figure $4 \mathrm{a}$, but now an external stress field, $\mathbf{S}^{0}$, is superimposed to the state of stress induced by the dislocation itself and given by (2.12). In the simplest case we make the hypothesis that $\mathbf{S}^{0}$ is constant and uniaxial, with the only non-zero component $\sigma_{22}^{0}$ in the $\mathbf{e}_{2}$ direction. Then, perform a perturbation of the dislocation tip of the same type of that represented in Figure $4 \mathrm{~b}$, with dislocation density $\rho\left(x_{1}\right)$ as per (2.13), under condition (2.14).

Following the same argument of (2.7) and (2.8), assuming a frictionless contact, the force exerted on the perturbed dislocation reads

$$
\bar{R}_{x 1}=-\int_{\xi_{1}}^{\xi_{2}}\left(\sigma_{22}\left(x_{1}, 0\right)+\sigma_{22}^{0}\right) \frac{\partial}{\partial x_{1}}\left(y_{2}\left(x_{1}, 0^{+}\right)-y_{2}\left(x_{1}, 0^{-}\right)\right) d x_{1} .
$$

However, from (2.18), the term associated with $\sigma_{22}\left(x_{1}, 0\right)$ is zero. Consequently, using (2.14) and (2.17), one finds that

$$
\bar{R}_{x 1}=-\sigma_{22}^{0} \int_{\xi_{1}}^{\xi_{2}} \frac{\partial}{\partial x_{1}}\left(y_{2}\left(x_{1}, 0^{+}\right)-y_{2}\left(x_{1}, 0^{-}\right)\right) d x_{1}=\sigma_{22}^{0} \int_{\xi_{1}}^{\xi_{2}} \rho\left(x_{1}\right) d x_{1}=\sigma_{22}^{0} b_{2} .
$$

This result is in agreement with the well-known Peach-Koehler formula, according to which the force on a dislocation of Burger's vector $\mathbf{b}$, submitted to a uniform stress field $\mathbf{S}^{0}$, results to be

$$
\mathbf{F}=\mathbf{S}^{0} \mathbf{b} \times \mathbf{e}_{3} .
$$

Formula (2.21) is obtained by considering $\mathbf{b}=b_{2} \mathbf{e}_{2}$, with $\mathbf{S}^{0}=\sigma_{22}^{0} \mathbf{e}_{2} \otimes \mathbf{e}_{2}$.

One can equivalently obtain the same result by a direct application of Elshelby's energy momentum tensor on the pillow path $\Gamma^{*}=\Gamma_{1}{ }^{*} \cup \Gamma_{2}{ }^{*} \cup \Gamma_{3}{ }^{*} \cup \Gamma_{4}{ }^{*}$ of the same type of that in Figure $2 \mathrm{c}$. The derivation is identical to that of (2.19).

Observe, again, that the result is independent of the particular perturbation that is assumed for the dislocation tip. Consequently, it holds, in the limit, when the original undistorted shape of the dislocation is recovered.

One could extend the same argument when the stress field $\mathbf{S}^{0}$ is not constant throughout the body, as it is the case, for example, in the evaluation of the interaction between two dislocations. Under this condition, one should consider a very small perturbation of the dislocation tip, with $\xi_{1}$ and $\xi_{2}$ infinitesimal quantities, very close to the zero value. If the stress $\mathbf{S}^{0}$ is regular in a neighborhood of the dislocation tip, then one can assume that the tip-perturbation can be choosen so small that in its neighborhood $\mathbf{S}^{0}$ can be considered constant in practice. In this case, the same argument that has led to (2.21) can be repeated. 


\subsubsection{Edge dislocation in a half plane}

With reference to Figure 5a, let $\Omega$ now represent the half plane $x_{1} \geq 0$ and consider an edge dislocation with Burger's vector $\mathbf{b}=b_{2} \mathbf{e}_{2}$, at a distance $\lambda$ from the plane $x_{1}=0$. Denoting with $r_{1}$ and $r_{2}$ the distances the generic point from the poles $(-\lambda, 0)$ and $(\lambda, 0)$, respectively, and indicating with $\theta_{1}$ and $\theta_{2}$ the angle formed by the corresponding radii with the $x_{1}$ axis, the corresponding Airy stress function reads (Dundurs, 1969)

$$
\chi=\frac{2 G b_{2}}{\pi(\kappa+1)}\left[r_{1} \log r_{1} \cos \theta_{1}-r_{2} \log r_{2} \cos \theta_{2}+\lambda\left(2 \log r_{2}-\cos \left(2 \theta_{2}\right)+\frac{2 \lambda}{r_{2}} \cos \theta_{2}\right)\right] .
$$

Making the substitutions $r_{1} \cos \theta_{1}=x_{1}-\lambda$ and $r_{2} \cos \theta_{2}=x_{1}+\lambda, r_{1}=\sqrt{\left(x_{1}-\lambda\right)^{2}+x_{2}^{2}}$ and $r_{2}=\sqrt{\left(x_{1}+\lambda\right)^{2}+x_{2}^{2}}$, the expression (2.23) reduces to

$$
\chi=\frac{2 G b_{2}}{\pi(\kappa+1)}\left[\frac{1}{2}\left(x_{1}-\lambda\right) \log \frac{\left(x_{1}-\lambda\right)^{2}+x_{2}^{2}}{\left(x_{1}+\lambda\right)^{2}+x_{2}^{2}}+\lambda \frac{\lambda^{2}-x_{1}^{2}+x_{2}^{3}}{\left(x_{1}+\lambda\right)^{2}+x_{2}^{2}}\right] .
$$

It is possible to calculate that the elastic strain energy associated with the dislocation is of the form (Dundurs, 1969)

$$
\Pi=\frac{G b_{2}^{2}}{\pi(\kappa+1)} \log \frac{\lambda}{\lambda_{0}}
$$

where $\lambda_{0}$ provides an irrelevant additive constant, having the dimension of length. Consequently, with a direct derivation analogous to (2.2), the configurational force exerted on the dislocation reads

$$
\hat{R}_{x 1}=-\frac{\partial \Pi}{\partial \lambda}=-\frac{G b_{2}^{2}}{\pi(\kappa+1) \lambda},
$$

whose negative sign means that the dislocation tends to be expelled from the body. 


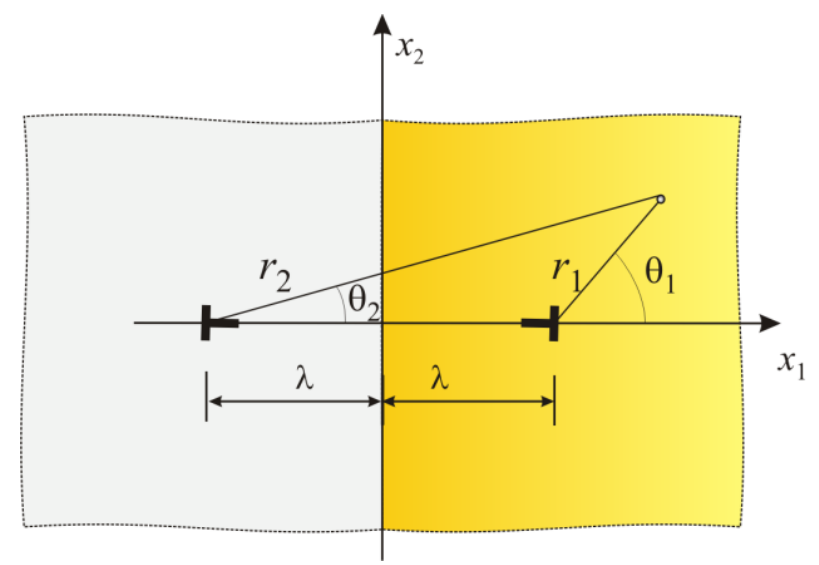

(a)

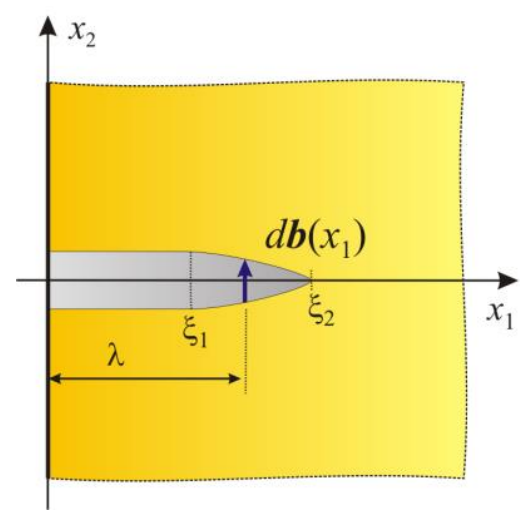

(b)

Figure 5. (a) The edge dislocation in semi-infinite medium. (b) The perturbed dislocation.

To apply the method of Section 2.1 it is required to evaluate the stress $\sigma_{22}\left(x_{1}, 0\right)$, which from $(2.24)$ reads

$$
\sigma_{22}\left(x_{1}, 0\right)=\frac{16 G b_{2}}{\pi(\kappa+1)} \frac{x_{1} \lambda^{2}}{\left(x_{1}-\lambda\right)\left(x_{1}+\lambda\right)^{3}} .
$$

Consider then a perturbation of the dislocation tip as per Figure 5b, of the same type of (2.13), under condition (2.14). Here, the interval $\left(\xi_{1}, \xi_{2}\right)$ is supposed to be in a small neighborhood of $x_{1}=\lambda$. We will assume that the perturbation is smooth enough, so that the state of stress becomes sufficiently regular to justify the mathematical derivation that follows. Then, from (2.27), the relevant component of stress associated with the perturbed configuration is

$$
\sigma_{22}\left(x_{1}, 0\right)=\frac{16 G}{\pi(1+\kappa)} \int_{\xi_{1}}^{\xi_{2}} \frac{x_{1} \xi^{2} \rho(\xi)}{\left(x_{1}-\xi\right)\left(x_{1}-\xi\right)^{3}} d \xi
$$

whereas the deformation jump on the line $x_{1}=0$ takes the same form (2.16). Then, recalling (2.17), with the same argument of (2.7) and (2.8), the force on the perturbed dislocation reads

$$
\bar{R}_{x 1}=-\int_{\xi_{1}}^{\xi_{2}} \sigma_{22}\left(x_{1}, 0\right) \frac{\partial}{\partial x_{1}}\left(y_{2}\left(x_{1}, 0^{+}\right)-y_{2}\left(x_{1}, 0^{-}\right)\right) d x_{1}=\frac{16 G}{\pi(1+\kappa)} \int_{\xi_{1}}^{\xi_{2}} \int_{\xi_{1}}^{\xi_{2}} \frac{x_{1} \xi^{2}}{\left(x_{1}-\xi\right)\left(x_{1}+\xi\right)^{3}} \rho(\xi) \rho\left(x_{1}\right) d \xi d x_{1} .
$$

However, using the fact that the crack tip is perturbed in a small neighborhood of $x_{1}=\lambda$, one can expand the above expression in Taylor's series under the hypothesis $\left|\xi_{1}-\lambda\right| / \lambda<<1$ and $\left|\xi_{2}-\lambda\right| / \lambda \ll<$. Then one finds 


$$
\frac{x_{1} \xi^{2}}{\left(x_{1}+\xi\right)^{3}}=\frac{\left[\lambda+\left(x_{1}-\lambda\right)\right][\lambda+(\xi-\lambda)]^{2}}{\left[2 \lambda+\left(x_{1}-\lambda\right)+(\xi-\lambda)\right]^{3}}=\frac{1}{8}+\frac{\xi-x_{1}}{16 \lambda}+o\left(\frac{x_{1}-\lambda}{\lambda}, \frac{\xi-\lambda}{\lambda}\right) \text {, }
$$

so that (2.29) becomes

$$
\begin{aligned}
R_{x 1} & =\frac{2 G}{\pi(1+\kappa)} \int_{\xi_{1}}^{\xi_{2}} \int_{\xi_{1}}^{\xi_{2}} \frac{\rho(\xi) \rho\left(x_{1}\right)}{\left(x_{1}-\xi\right)} d \xi d x_{1}-\frac{G}{\pi(1+\kappa) \lambda} \int_{\xi_{1}}^{\xi_{2}} \int_{\xi_{1}}^{\xi_{2}} \rho(\xi) \rho\left(x_{1}\right) d \xi d x_{1}+O\left(\frac{x_{1}-\lambda}{\lambda}, \frac{\xi-\lambda}{\lambda}\right) \\
& =-\frac{G b_{2}^{2}}{\pi(1+\kappa) \lambda}+O\left(\frac{x_{1}-\lambda}{\lambda}, \frac{\xi-\lambda}{\lambda}\right) .
\end{aligned}
$$

In fact, the first integral in (2.31) vanishes because the integration domain is symmetric with the respect to the line $x_{1}=\xi$, while the integrand is antisymmetric, whereas the value of the second integral directly comes from (2.14).

The same quantity of (2.26) is obtained in the limit $\left(\xi_{1}-\lambda\right) / \lambda \rightarrow 0$ and $\left(\xi_{2}-\lambda\right) / \lambda \rightarrow 0$. In other words, the classic expression for the configurational forces is obtained via simple equilibrium only in the limit when the original undistorted configuration is restored. This is a consequence of the fact that, contrary to the cases analyzed in Sections 2.2.1 and 2.2.2, there is now a length scale in this problem. This is represented by the distance $\lambda$ between the tip of the dislocation and the free boundary: the perturbation of the crack tip must be small with respect to $\lambda$ in order to recover the classical formulas.

Finally, observe that the same derivation of the configurational force could be considered, similarly to (2.11), with the direct application of Elshelby's energy momentum tensor on the pillow path $\Gamma^{*}=\Gamma_{1}{ }^{*} \cup \Gamma_{2}{ }^{*} \cup$ $\Gamma_{3}{ }^{*} \cup \Gamma_{4}{ }^{*}$, of the same type of that in Figure 2c.

\section{CONFIGURATIONAL FORCES ON CRACKS}

The Newtonian representation of the configurational forces acting on fractures can be obtained by performing an appropriate perturbation that, in our interpretation, consists in assuming that the relative displacement between crack surfaces are resisted by cohesive forces à la Barenblatt. The original unperturbed configuration of the crack is recovered in the small-scale bridging limit. An additional difficulty with respect to the previous Section is that, whereas a dislocation can be physically visualized by an equivalent inclusion, as an extra material-slice modifying the natural state of the body, the crack tip, where the classical configurational forces are supposed to be applied, is somehow immaterial. In our interpretation the configurational forces should be considered applied to an object of substance that comprises the ligaments that bridge the crack tip, ligaments that in the small scale bridging limit converge towards the crack tip.

To illustrate this idea, it is useful to consider first a simplified model of crack with a double beam layout, 
propaedeutic for the study of the general case.

\subsection{The double beam layout}

A particular double-beam arrangement is represented in Figure 6a. Two identical elastic beams are connected along a part of their length by a set of "roller-clamps", each one formed by two rollers joined by springs. These are the devices that keep the two beams together: the upper roller is in contact with extrados of the upper beam, while the lower roller touches the intrados of the lower beam. Let $a$ represent the radius of curvature of the rollers and let $K$ denote the (non-necessarily constant) stiffness of the springs.

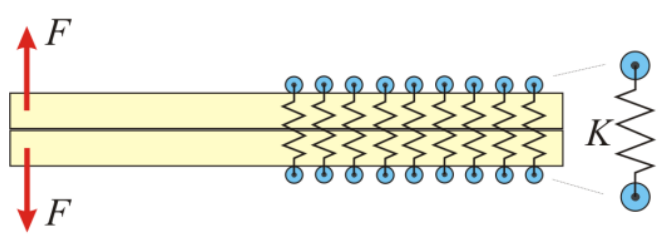

(a)

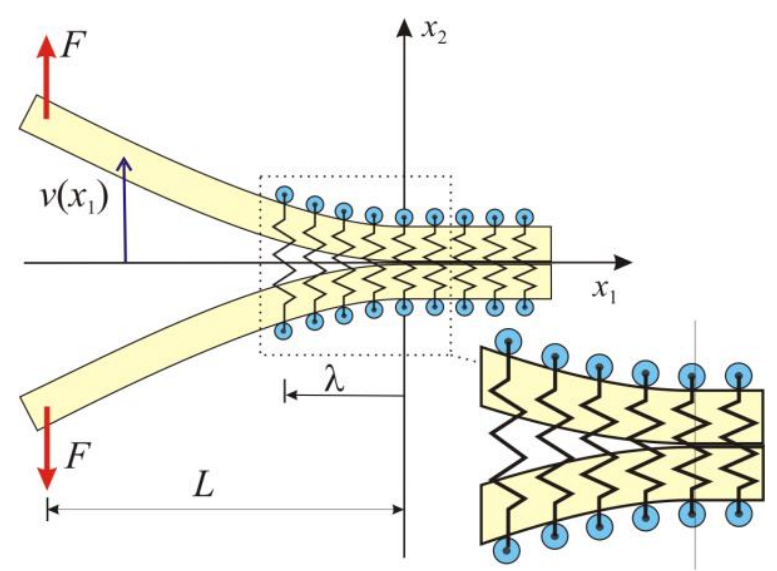

(b)

Figure 6. (a) The elementary double beam layout model. (b) Contact problem for the beam layout.

The action of two opposite forces $F$, applied at the left ends, produces the relative displacement of the beams, as represented in Figure 6b. Clearly, only one portion of interface of the two beams will remain in contact, whereas there will be a detachment in the complementary portion. Introduce the reference system $\left(x_{1}, x_{2}\right)$ as in Figure 6b, with the origin at the frontier point between the adherent and the detached regions of the interface. Let then $\lambda$ represent the distance from the origin of the latest roller clamp on the left-hand-side, so that the zone where the springs of the clamps are tensioned is $-\lambda<x_{1}<0$. On the other hand, the deformation of the beam is null and the contact forces vanish on $x_{1}<-\lambda$.

Symmetry allows consideration of equilibrium of but the upper beam. Let $v\left(x_{1}\right)$ represent the displacement in the $x_{2}$ direction of the centroid of the cross section that is at $x_{1}$ in the undistorted configuration. Indicate with $E$ and $I$ the Young's modulus and moment of inertia of the beam, for which we assume the EulerBernoulli model. Therefore, under the hypothesis that $\left|v^{\prime}\left(x_{1}\right)\right|<<1$, the bending moment $M\left(x_{1}\right)$ is related with the second derivative of the displacement field $v^{\prime \prime}\left(x_{1}\right)$ according to the constitutive equation $M\left(x_{1}\right)=E I v^{\prime \prime}\left(x_{1}\right)$. 
Concerning the radius $a$ of the roller (see Figure 6a), in the following we will assume that $a<<\left|1 / v^{\prime \prime}\left(x_{1}\right)\right|$.

In the limit in which the clamps are rigid, i.e., when $K \rightarrow \infty$, then $\lambda=0$. In this case, the contact forces between the beams present a strong singularity, since they are equipollent to a concentrated force equal to $F$ plus a dipole, i.e., a double force with moment, applied at $x_{1}=0$, whose strength is required to equilibrate the bending moment $M(0)=F L$. All the roller clamps are stress-free, apart from the one at $x_{1}=0$, which provides the forces necessary for equilibrium. The free body diagram of a beam portion in a neighborhood of $x_{1}=0$ is represented in Figure 7a, in which the equilibrium configuration corresponds to the limit $\varepsilon \rightarrow 0$.

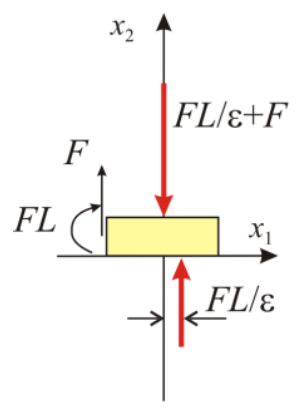

(a)

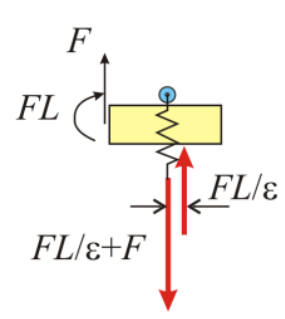

(b)
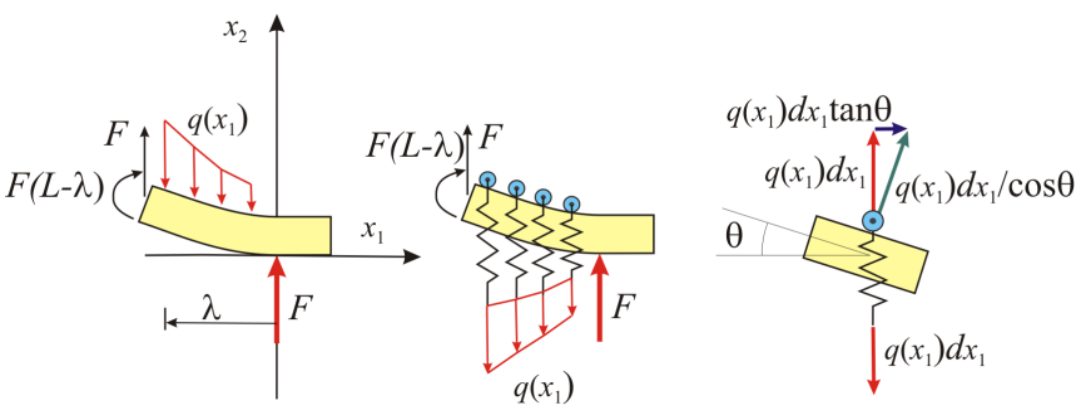

Figure 7. Free body diagram of a portion of the upper beam in a neighborhood of $x_{1}=0$ in the case of (a) rigid clamps $K \rightarrow \infty$, and (b) elastic clamps.

What should be observed is that, in this condition, the horizontal force on the clamp is null because $v^{\prime}(0)=0$. However, one can demonstrate that a configurational force acts on the clamp in the positive $x_{1}$ direction. A direct application of Clapeyron's theorem shows in fact that the total strain energy associated with the beam deflection is $-\frac{1}{2} F^{2} L^{3} / 3 E I$. If the clamp is displaced in the positive $x_{1}$ direction of the quantity $\xi$, the total energy of each beam reads

$$
\Pi=-2 \frac{1}{2} \frac{F^{2}(L+\xi)^{3}}{3 E I},
$$

so that the configurational force reads

$$
\hat{R}_{x 1}=-\left.\frac{\partial \Pi}{\partial \xi}\right|_{\xi=0}=\frac{F^{2} L^{2}}{E I}>0 .
$$

This is the horizontal force that is exerted on the rigid roller clamp at $x_{1}=0$. Such a contribution has been obtained by means of an energetic argument. It is difficult, however, to visualize such a force using pure statics. In order to solve this apparent paradox, a perturbation is made by relaxing the hypothesis of rigid 
clamps. When the clamps are elastic, the situation is the one represented in Figure $7 \mathrm{~b}$. Now the springs provide the action to equilibrate the effects of the force $F$ and of the bending moment $F(L-\lambda)$ at $x_{1}=-\lambda$. Under the hypothesis that the clamps are numerous, one can take a smeared view of the reaction forces due to the springs and assume they can be described by the force per unit length $q\left(x_{1}\right)$, for $-\lambda<x_{1}<0$, whose magnitude is a function of the displacement field $v\left(x_{1}\right)$. A concentrated contact force $F^{*}$ is acting at $x_{1}=0$, whose magnitude is determined from the equilibrium in the $x_{2}$ direction. Compatibility with the beam deflection obviously requires that $E I V^{\prime \prime \prime \prime}\left(x_{1}\right)=-q\left(x_{1}\right)$ for $-\lambda<x_{1}<0$.

Notice that, contrary to the case $K \rightarrow \infty$, now there is a non-null horizontal forces on the active clamps. In fact, assuming a frictionless contact, the reaction exerted by the beam on the roller can only take the direction of the normal to the deformed axis, forming an angle $\theta$ with respect to the $x_{2}$ axis, with $\tan \theta=-v^{\prime}\left(x_{1}\right)$. Such a contribution is $d H=q\left(x_{1}\right) \tan \theta d x_{1}=-q\left(x_{1}\right) v^{\prime}\left(x_{1}\right) d x_{1}$. Taking into account that an identical contribution is provided by the lower beam on the same roller clamp, the resultant of all the horizontal forces acting on the active clamps results to be

$$
\bar{R}_{x 1}=-2 \int_{0}^{\lambda} q\left(x_{1}\right) v^{\prime}\left(x_{1}\right) d x_{1}=2 E I \int_{0}^{\lambda} v^{\prime \prime \prime \prime}\left(x_{1}\right) v^{\prime}\left(x_{1}\right) d x_{1}
$$

Now, recall that $M\left(x_{1}\right)=E I v^{\prime \prime}\left(x_{1}\right)$, with $M(0)=0$ and $M(-\lambda)=F(L-\lambda)$; moreover, $V\left(x_{1}\right)=-M^{\prime}\left(x_{1}\right)=-E I v^{\prime \prime \prime}\left(x_{1}\right)$, with $V(-\lambda)=-F$. Since

$$
\begin{aligned}
& \int_{0}^{\lambda} v^{\prime \prime \prime \prime}\left(x_{1}\right) v^{\prime}\left(x_{1}\right) d x_{1}=\left[v^{\prime \prime \prime}\left(x_{1}\right) v^{\prime}\left(x_{1}\right)\right]_{0}^{\lambda}-\int_{0}^{\lambda} v^{\prime \prime \prime}\left(x_{1}\right) v^{\prime \prime}\left(x_{1}\right) d x_{1} \\
& \int_{0}^{\lambda} v^{\prime \prime \prime}\left(x_{1}\right) v^{\prime \prime}\left(x_{1}\right) d x_{1}=\left[v^{\prime \prime}\left(x_{1}\right) v^{\prime \prime}\left(x_{1}\right)\right]_{0}^{\lambda}-\int_{0}^{\lambda} v^{\prime \prime \prime}\left(x_{1}\right) v^{\prime \prime}\left(x_{1}\right) d x_{1}
\end{aligned}
$$

recalling that $v^{\prime}(0)=0$, from $(3.3)$ one can write

$$
\bar{R}_{x 1}=2 E I\left\{\left[v^{\prime \prime \prime}\left(x_{1}\right) v^{\prime}\left(x_{1}\right)\right]_{-\lambda}^{0}-\frac{1}{2}\left[v^{\prime \prime}\left(x_{1}\right) v^{\prime \prime}\left(x_{1}\right)\right]_{-\lambda}^{0}\right\}=-F v^{\prime}(-\lambda)+\frac{F^{2}(L-\lambda)^{2}}{E I} .
$$

In the limit for $\lambda \rightarrow 0$, since also $v^{\prime}(-\lambda) \rightarrow 0$, one finds from (3.5) the same expression (3.2).

The aforementioned derivation holds for any assumed constitutive equation for the springs that bind the rollers of the clamps. In particular, the limit value of (3.5) for $\lambda \rightarrow 0$ is completely independent from the particular stiffness of the springs and, specifically, it applies in the borderline case of springs with infinite stiffness. In this condition, the angle $\theta$ represented in Figure $7 \mathrm{~b}$ tends to become zero, but at the same time the force per unit length $q\left(x_{1}\right)$ tends to become infinite, so that a non-zero limit for the horizontal component can be attained. 
In conclusion, we have provided a direct "Newtonian" derivation of the configurational forces that, in the simplified model here presented, act on the set of clamps that result to be active in maintaining the two beams into contact.

\subsection{Configurational forces on straight cracks opening in mode-I.}

Consider the plane problem of a straight crack opening in mode-I. Being interested in the stress and deformation fields in a neighborhood of the crack tip, we may consider the asymptotic solution of a semiinfinite crack traversing an infinite, homogeneous and isotropic, elastic medium, with shear modulus $G$, Poisson's ratio $v$ and Young's modulus $E=2 G(1+v)$. What is important for the following considerations, is determining the deformation of the crack surfaces consequent to the applied external loads. It is well known that such deformation can be equivalently calculated by assuming that the crack is opened by a pressure $p\left(x_{1}\right)$, matching the effects of the external actions. We will follow this approach for the sake of mathematical convenience.

Let the crack occupy the position $x_{2}=0, x_{1} \leq 0$ and assume plane strain conditions. Near the crack tip the stress field component $\sigma_{22}\left(x_{1}, 0\right), x_{1}>0$, and the displacement component $u_{2}\left(x_{1}, 0^{ \pm}\right), x_{1}<0$, are of the form

$$
\sigma_{22}\left(x_{1}, 0\right)=\frac{K_{I}}{\sqrt{2 \pi x_{1}}}+O(1), \quad x_{1}>0 ; \quad u_{2}\left(x_{1}, \pm 0\right)= \pm \frac{2(1-v)}{G} K_{I} \sqrt{\frac{-x_{1}}{2 \pi}}+O\left(-x_{1}\right)^{3 / 2}, x_{1}<0,
$$

where $K_{I}$ is the stress intensity factor in mode $I$, determined as

$$
K_{I}=\sqrt{\frac{2}{\pi}} \int_{-\infty}^{0} \frac{p\left(x_{1}\right)}{\sqrt{-x_{1}}} d x_{1}
$$

The integral (3.7) is supposed to be finite, i.e., the function $p\left(x_{1}\right)$ has a sufficiently fast decay for $x_{1} \rightarrow-\infty$. Without loss of generality, we will suppose that $p\left(x_{1}\right)$ is a regular and bounded function, with $p\left(x_{1}\right) \cong 0$ for $x_{1} \leq$ $-L$, and $p\left(x_{1}\right) \leq P_{M}<\infty$ in $-L \leq x_{1} \leq 0$.

\subsubsection{Direct energetic derivation}

If the cracks propagates of $\xi$ in the $x_{1}$ direction, with $\xi$ small, one has that the new crack opening $\delta u_{2}\left(x_{1}, \pm 0\right)$ reads

$$
\delta u_{2}\left(x_{1}, \pm 0\right)=\sqrt{\frac{2}{\pi}} \frac{(1-v)}{G} K_{I} \sqrt{-x_{1}+\xi}+O(\xi)^{3 / 2}, x_{1}<\xi
$$


so that the corresponding variation of the total energy, up to first order in $\xi$, reads

$$
\Delta \Pi=-\frac{1}{2} \int_{0}^{\xi} \sigma_{22}\left(x_{1}, 0\right) 2 \delta u_{2}\left(x_{1},+0\right) d x_{1}=-\frac{(1-v)}{\pi G} K_{I}^{2} \int_{0}^{\xi}\left(\frac{-x_{1}+\xi}{x_{1}}\right)^{1 / 2} d x_{1}=-\frac{(1-v)}{2 G} K_{I}^{2} \xi
$$

Consequently, there is a configurational force that drives the crack to extend. Such a force, from an energetic argument, takes the form

$$
\hat{R}_{x 1}=-\frac{\partial \Delta \Pi}{\partial \xi}=\frac{(1-v)}{2 G} K_{I}^{2}
$$

The force of (3.10) cannot be visualized from pure statics because, in the elastic solution, every reference part of the body is in equilibrium.

\subsubsection{Newtonian interpretation. The cohesive block}

It is then necessary to make a perturbation similar in type to that used in Section 3.1 for the case of the double beam arrangement. Imagine then a slight modification of the configuration of the sharp crack, by assuming that the crack lips are bridged by forces of attraction $a ̀$ la Barenblatt. At this stage, it is not essential to define how such forces should derive from some law of cohesion, because we are just interested in a perturbation of the elastic solution with the aim of relaxing the singularity at the crack tip. However, in agreement with physical intuition, such cohesive forces should be a function of the crack opening displacement: they increase up to a critical separation, beyond which they tend to decrease and become null if the separation is large enough so that, at this stage, the corresponding portion of the crack surface is stressfree.

The cohesive (traction) forces per unit area will be indicated with $q\left(x_{1}\right)$ and they obey to some constitutive equation of the type

$$
q\left(x_{1}\right)=f\left[u_{2}\left(x_{1}, 0^{+}\right)-u_{2}\left(x_{1}, 0^{-}\right)\right]=f\left[2 u_{2}\left(x_{1}, 0^{+}\right)\right],
$$

where we have used the fact that crack opening is symmetrical. In general, the function $f$ is supposed to be regular, with $f[2 v]=0$ for $2 v \geq 2 v_{0}$ and a maximum $f_{M}$ of the same order of the material elastic modulus, i.e, $f_{M}=O(G)$.

In a neighborhood of the crack tip there will be an edge region $-\lambda \leq x_{1} \leq 0$, with $\lambda<L$, where the crack opening displacement is less than $2 v_{0}$, compatibly with the presence of cohesive forces, whereas on the complementary part $x_{1} \leq-\lambda$ the cohesive forces are negligible. This is in agreement with the classical 
hypotheses by Barenblatt (Barenblatt, 1962).

The region where the cohesive forces are active can be visualized with the naked eye in an experiment with a very soft material (for harder materials a magnifying device is usually needed). To illustrate, Figure $8 \mathrm{a}$ represents a crack forming in a hydrogel, propagating in horizontal direction. Here, the cohesive forces are concentrated in an object of substance, approximately triangular-shaped, in the sequel referred to as the "cohesive block", which can be distinguished because here the strain is much higher than in the rest of the body. The crack profile is recognizable in the picture, as well as the interface that delimits the cohesive block from the remaining part of the body.

The cohesive block can be imagined to be nucleated from a very thin interface layer in contact with the crack lips in a neighborhood of the tip, which expands due to the very high strain undergone in that region. One can consider the borderline case in which the thickness of such layer is infinitesimal, so that the cohesive block represents a volume that is "created" from a surface, and that remains in contact with the lips of the opening crack. This limit-case view is somehow specular to what traditionally assumed for cohesive forces $\grave{a}$ la Barenblatt, if one thinks that they are associated with the reaction of springs of initially evanescent thickness, delimited by the gap of the undistorted fracture. This discussion about the supposed nature of the cohesive block is of importance because, in the following derivations, the configurational forces will be supposed to be applied on the cohesive block itself.

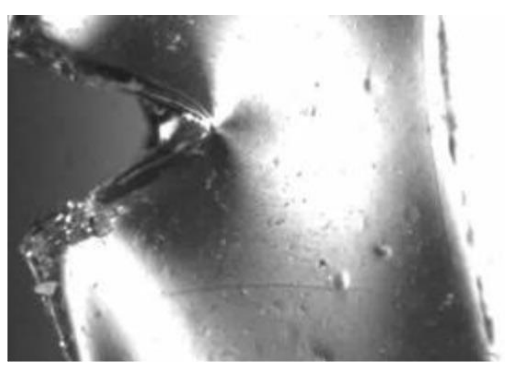

(a)
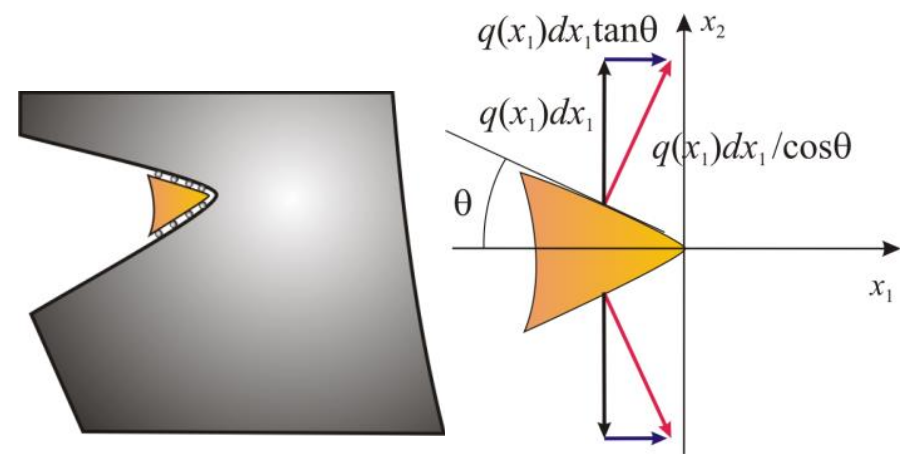

(b)

Figure 8. (a) Fracture of a hydrogel, with evidence of the cohesive block (from https://www.simm.espci.fr/spip.php?article250\&lang=fr). (b) Schematic of the stress block and and free body diagram.

What we want to calculate now is the resultant of the shear component of the contact forces that are applied along the boundary of the cohesive block by the remaining part of the body. Such forces must be calculated taking into account the deformation of the body, in particular the inclination of the crack surfaces with respect to the undistorted crack profile.

To do so, as qualitatively represented on the left-hand-side picture of Figure $8 \mathrm{~b}$, imagine that the 
cohesive block is constrained by a set of rollers to the crack surfaces. Such rollers are supposed to be bilateral (they can withstand tensile stresses), and they can provide constraint reactions only in the direction of the normal to the (deformed) crack surface. The free-body diagram of the cohesive block is represented on the right-hand-side of Figure $8 \mathrm{~b}$. The forces acting on an elementary portion of the interface, presenting $d x_{1}$ as horizontal projection, must provide the cohesive forces that are needed to bridge the crack surfaces. Therefore, the vertical component of such forces is $q\left(x_{1}\right) d x_{1}$. However, denoting with $\theta$ denotes the angle that the tangent to the interface forms with the horizontal, there is an unbalanced horizontal component equal to $q\left(x_{1}\right) d x_{1} \tan \theta$. The resultant on this latter contributions on the whole interface, provides an unbalanced force, say $\bar{R}_{x 1}$, that pushes the cohesive block towards the positive direction of the $x_{1}$ axis, and that can only be equilibrated by the shear contact forces at the interface. If no shear force is present at the interface, the cohesive block would be pushed by the force $\bar{R}_{x 1}$ towards the positive direction of the axis $x_{1}$, producing a wedging action for the crack. This is the counterpart, for the continuum case, of the horizontal forces on the elastic clamps represented in Figure $7 \mathrm{~b}$ for the double beam layout.

Obviously, if one neglects the deformation of the crack lips, the force component $\bar{R}_{x 1}$ is negligible because the undistorted crack is straight and $\theta=0$. Indeed, such a force is a result of second order geometric effects. It would have been perhaps more rigorous to solve the crack problem within the framework of nonlinear elasticity theory, taking particularly into account geometric non-linearities, but this would involve formidable analytical difficulties. However, we will show in the following that the argument pursued so far, which considers the linear-elasticity equations in the undistorted state and takes into account the deformation a-posteriori, can be regarded as a second order approximation that provides consistent results, whose accuracy increases with decreasing angle $\theta$.

In conclusion, since $\tan \theta=-\frac{\partial}{\partial x_{1}} u_{2}\left(x_{1}, 0^{+}\right)=\frac{\partial}{\partial x_{1}} u_{2}\left(x_{1}, 0^{-}\right)$, summing up the contributions on the two crack lips, one finds that

$$
\bar{R}_{x 1}=-\int_{-\lambda}^{0} q\left(x_{1}\right) \frac{\partial}{\partial x_{1}}\left(u_{2}\left(x_{1}, 0^{+}\right)-u_{2}\left(x_{1}, 0^{-}\right)\right) d x_{1}=-2 \int_{-\lambda}^{0} q\left(x_{1}\right) \frac{\partial}{\partial x_{1}}\left(u_{2}\left(x_{1}, 0^{+}\right)\right) d x_{1} .
$$

This expression coincides with that proposed by Rice in (Rice, 1968) for the evaluation of the of the strain energy release rate in a Barenblatt-Dugdale crack model by a direct application of the J-integral.

Now, following the classical argument by Barenblatt (Barenblatt, 1962), the stress at the crack tip must be finite, i.e., the cohesive forces must adjust themselves so that they reduce to zero the stress intensity factor. This condition, from (3.7), can be stated as 


$$
\int_{-\infty}^{0} \frac{p\left(x_{1}\right)}{\sqrt{-x_{1}}} d x_{1}+\int_{-\infty}^{0} \frac{-q\left(x_{1}\right)}{\sqrt{-x_{1}}} d x_{1}=0
$$

where we have implicitly indicated that $q\left(x_{1}\right)=0$ for $x_{1}<-\lambda$. On the other hand, we know that

$$
u_{2}\left(x_{1}, 0^{ \pm}\right)= \pm \int_{-\infty}^{0}(p(\tau)-q(\tau)) m\left(x_{1}, \tau\right) d \tau, \quad \text { with } m\left(x_{1}, \tau\right)=\frac{1-v}{\pi G} \log \frac{\sqrt{-x_{1}}+\sqrt{-\tau}}{\left|\sqrt{-x_{1}}-\sqrt{-\tau}\right|}
$$

Consequently, evaluating the integrals in the sense of Cauchy principal value, one finds that

$$
\begin{gathered}
\frac{\pi G}{1-v} \frac{\partial}{\partial x_{1}}\left(u_{2}\left(x_{1}, 0^{+}\right)\right)=\frac{1}{\sqrt{-x_{1}}} \int_{-\infty}^{0} \frac{(p(\tau)-q(\tau)) \sqrt{-\tau}}{\tau-x_{1}} d \tau=\frac{1}{\sqrt{-x_{1}}} \int_{-\infty}^{0}(p(\tau)-q(\tau))\left(\frac{\sqrt{-\tau}}{\tau-x_{1}}+\frac{1}{\sqrt{-\tau}}-\frac{1}{\sqrt{-\tau}}\right) d \tau \\
=\frac{1}{\sqrt{-x_{1}}} \int_{-\infty}^{0}(p(\tau)-q(\tau))\left(\frac{-x_{1}}{\left(\tau-x_{1}\right) \sqrt{-\tau}}-\frac{1}{\sqrt{-\tau}}\right) d \tau=\sqrt{-x_{1}} \int_{-\infty}^{0} \frac{p(\tau)-q(\tau)}{\left(\tau-x_{1}\right) \sqrt{-\tau}} d \tau,
\end{gathered}
$$

where, following an argument similar to that proposed in (Willis, 1967), we have used (3.13). But, since the integral in (3.7) has to be finite and recalling (3.13), one has that

$$
\frac{1-v}{\pi G} \sqrt{-x_{1}} \int_{-\infty}^{0} \frac{p(\tau)}{\left(\tau-x_{1}\right) \sqrt{-\tau}} d \tau=O\left[\frac{P_{M}}{G}\right], \quad \frac{1-v}{\pi G} \sqrt{-x_{1}} \int_{-\infty}^{0} \frac{q(\tau)}{\left(\tau-x_{1}\right) \sqrt{-\tau}} d \tau=O\left[\frac{f_{M}}{G}\right]=O[1] .
$$

Therefore, since $P_{M} \ll f_{M}$, in (3.16) one can neglect the term in $p\left(x_{1}\right)$ with respect to that in $q\left(x_{1}\right)$, so that (3.12) reduces to

$$
\bar{R}_{x 1}=2 \frac{1-v}{\pi G} \int_{-\lambda}^{0} q\left(x_{1}\right)\left[\sqrt{-x_{1}} \int_{-\lambda}^{0} \frac{q(\tau)}{\left(\tau-x_{1}\right) \sqrt{-\tau}} d \tau\right] d x_{1}\left\{1+O\left[\frac{P_{M}}{f_{M}}\right]\right\}
$$

Interchanging $x_{1}$ and $\tau$, one finds

$$
\bar{R}_{x 1}=2 \frac{1-v}{\pi G} \int_{-\lambda}^{0} q(\tau)\left[\sqrt{-\tau} \int_{-\lambda}^{0} \frac{-q\left(x_{1}\right)}{\left(\tau-x_{1}\right) \sqrt{-x_{1}}} d x_{1}\right] d \tau\left\{1+O\left[\frac{P_{M}}{f_{M}}\right]\right\}
$$

so that, summing up, one obtains

$$
\bar{R}_{x 1}=2 \frac{1-v}{\pi G} \int_{-\lambda}^{0} \frac{q(\tau)}{\sqrt{-\tau}} d \tau \int_{-\lambda}^{0} \frac{q\left(x_{1}\right)}{\sqrt{-x_{1}}} d x_{1}\left\{1+O\left[\frac{P_{M}}{f_{M}}\right]\right\}
$$

Finally, recalling from (3.7) and (3.13) that 


$$
\int_{-\lambda}^{0} \frac{q(\tau)}{\sqrt{-\tau}} d \tau=\sqrt{\frac{\pi}{2}} K_{I}
$$

the final result is that

$$
\bar{R}_{x 1}=\frac{1-v}{2 G} K_{I}^{2}\left\{1+O\left[\frac{P_{M}}{f_{M}}\right]\right\}
$$

As the cohesive forces increase in magnitude, the region where they must be active to annihilate the stress intensity factor becomes smaller. Therefore, the small scale bridging limit $\lambda \rightarrow 0$ is attained when $f_{M} \rightarrow+\infty$. In this limit, the formula (3.21) coincides with that for the energy-derived expression $\hat{R}_{x 1}$ of (3.10). The result is independent of the particular assumed distribution of cohesive forces at the crack tip. Indeed, it is not even necessary to assume that they are derivable from a cohesive law of the type (3.11), because their only important property is that, while being localized in a very small neighborhood of the crack tip, they can regularize the stress singularity as per (3.13).

A question arises: where is the configurational force applied? Progressing in the small scale bridging limit, the cohesive block of Figure $8 \mathrm{~b}$ tends to shrink and flattens towards the crack tip. In the limit, one may imagine that the block becomes a material point, which embraces all the interatomic forces that prevent the material from fracturing and occupies the same position of the crack tip. This is our physical interpretation of the common statement that the configurational force is concentrated and applied at the crack tip (Gurtin \& Podio-Guidugli, 1996).

\subsubsection{Energetic versus configurational balance}

Although the argument is standard, it may be worth to recall that the same expression (3.12) coincides with that obtainable by accounting for the energy dissipated in the cohesive block. Suppose that under the applied pressure $p\left(x_{1}\right)$ the crack is just about to propagate so that (3.11) holds with $u_{2}\left(-\lambda, 0^{+}\right)-u_{2}\left(-\lambda, 0^{-}\right)=2 v_{0}$. If the crack tip moves towards the positive $x_{1}$ direction of $\xi$, then a new equilibrium state will be attained under a new distribution of cohesive forces. In particular, one expects that new cohesive forces will be nucleated in $0<x_{1}<\xi$, and the old cohesive forces will be annihilated in $-\lambda<x_{1}<-\lambda+\xi$. If the length of the cohesive zone $\lambda$ is much smaller than the reference crack length, and if $\xi \ll \lambda$, the shape of the crack tip will remain unchanged. Consequently, the new crack opening displacement will be given by $u_{2}\left(x_{1}-\xi, 0^{+}\right)-u_{2}\left(x_{1}-\xi, 0^{-}\right)$.

Let us then define the potential of the cohesive forces per unit area as 


$$
F[s]=\int_{0}^{s} f[\tau] d \tau
$$

Since crack opening is symmetric, the energetic variation of the cohesive forces included in the stress block, associated with the movement of the crack, is

$$
\Delta \Lambda(\xi)=\int_{-\lambda}^{\xi} F\left[2 u_{2}\left(x_{1}-\xi, 0^{+}\right)\right]-F\left[2 u_{2}\left(x_{1}, 0^{+}\right)\right] d x_{1},
$$

and the rate of change of such term at $\xi=0$ reads

$$
\begin{aligned}
& \left.\frac{d}{d \xi}(\Delta \Lambda(\xi))\right|_{\xi=0} \\
& \quad=\left\{F\left[2 u_{2}\left(x_{1}-\xi, 0^{+}\right)\right]-F\left[2 u_{2}\left(x_{1}, 0^{+}\right)\right]+\int_{-\lambda}^{\xi}-f\left[2 u_{2}\left(x_{1}-\xi, 0^{+}\right)\right] \frac{\partial}{\partial x_{1}}\left(2 u_{2}\left(x_{1}-\xi, 0^{+}\right)\right) d x_{1}\right\}_{\xi=0} \\
& \quad=\int_{-\lambda}^{0}-f\left[2 u_{2}\left(x_{1}, 0^{+}\right)\right] \frac{\partial}{\partial x_{1}}\left(2 u_{2}\left(x_{1}, 0^{+}\right)\right) d x_{1}=-2 \int_{-\lambda}^{0} q\left(x_{1}\right) \frac{\partial}{\partial x_{1}}\left(u_{2}\left(x_{1}, 0^{+}\right)\right) d x_{1} .
\end{aligned}
$$

Remarkably, one has

$$
\int_{-\lambda}^{0}-f\left[2 u_{2}\left(x_{1}, 0^{+}\right)\right] \frac{\partial}{\partial x_{1}}\left(2 u_{2}\left(x_{1}, 0^{+}\right)\right) d x_{1}=\int_{2 v_{0}}^{0}-f\left[2 u_{2}\left(x_{1}, 0^{+}\right)\right] d\left(2 u_{2}\left(x_{1}, 0^{+}\right)\right)=\int_{0}^{2 v_{0}} f[\tau] d \tau=\int_{0}^{\infty} f[\tau] d \tau .
$$

The latter expression represents the energy per unit area that has to be expended to overcome the cohesive forces and produce the material separation. Following Griffith, this is the surface energy $2 \gamma$.

The energetic competition between energy release rate and fracture energy can acquire a different interpretation from the "Newtonian" point of view. Observing the free body diagram of the cohesive block represented in Figure 8b, one may argue that the driving force is produced through the sliding of the interface, whereas the restoring force is associated with the actions that oppose to such sliding. The former is the configurational force, while the latter is represented by a force that, in this interpretation, plays somehow the role of "shear cohesion".

In the small-scale bridging limit the cohesive block tends to shrink onto the crack tip. In this case, the driving force tends to be proportional to the second power of the stress intensity factor, whereas the restoring force can be represented by $2 \gamma$. Therefore the most celebrated relationship

$$
\hat{R}_{x 1}=\frac{(1-v)}{2 G} K_{I}^{2}=2 \gamma,
$$


represents the balance of linear momentum, in the direction of crack propagation, for a material point, coinciding with the crack tip, which represents the small-scale-bridging limit of the cohesive block.

\section{TOWARDS A GENERALIZATION}

The problems presented in the previous sections are illustrative examples, but the Newtonian interpretation of configurational forces can be addressed in a general setting. Since at the base of the approach there is the balance of linear momentum for a definite material region, one has first to establish an analogy between the notion of "defect", which in general represents a state of disordered material structure, and an object of substance, to which Newtonian forces can be applied. Another key point is the definition of the nature of the contact between such an object and the surrounding matrix. To illustrate, the forthcoming deductions are specialized to the paradigmatic case of a general Somigliana dislocation.

\subsection{The Somigliana dislocation}

The equivalence between a "defect" and an "object of substance" is at the base of the seminal work by Eshelby (Eshelby, 1951), who treated the defect as a form of material inclusion: the body is cut and material substance is inserted in the cut by forcing inside some new extra material ${ }^{2}$ and afterwards everything is welded together. The inclusion is not necessarily rigid, because the pasted material can be the same as that of the pristine body; no inhomogeneity is thus created. An eigenstress state is produced inside the body, and the extra-substance may represent the object where the Newtonian counterparts of configurational forces can be thought to be applied.

It is convenient to refer explicitly to the ideal procedure proposed at the beginning of the last century by Carlo Somigliana. Consider a generic surface $\omega$ either internal or touching the boundary of the body $\Omega \subset \mathbb{R}^{3}$, supposed undistorted and stress free in this reference configuration. Make a cut over $\omega$ and give to the resulting faces of the cut a small relative displacement. The remaining gap is filled with new material and welded together is such a way that the relative displacement, previously imposed, remains unaltered after that the cut is released. This could be certainly be obtained if the pasted material is rigid, but the result is analogous if the material is the same as that of the matrix, provided that it is prestressed by the same forces that are necessary to open the cut so that, after welding, equilibrium can be reached with no additional deformation. After this

\footnotetext{
${ }^{2}$ One can obtain an eigenstress state also by removing, rather than adding, material. The considerations that follow may be applied also to this case, but since the forcing of extra material is physically more intuitive, reference will be explicitly made to this condition only.
} 
operation, the internal strain is completely characterized by assigning the vectorial function $\mathbf{b}(\mathbf{x}): \omega \rightarrow \mathbb{R}^{3}$, later referred to as the Burger's vector, which indicates the final separation of points on opposite sides of the cut, originally coinciding on $\omega$.

If $\mathbf{b}(\mathbf{x})$ is reasonably smooth, the state of stress should be finite everywhere, except possibly at some edge points. A particular class of Somigliana dislocations, referred to by Eshelby as physical dislocations (Eshelby, 1951), is that for which $\mathbf{b}(\mathbf{x})$ is constant on $\omega$. A number of dislocation loops laying in a surface can be regarded as Somigliana dislocations for which $\mathbf{b}(\mathbf{x})$ is a stepped function, but this produces in general stress singularities in a neighborhood of the borders where the step-discontinuity is imposed. However, at a distance that is large with respect to the spacing of the individual dislocation, the stress is equivalent to that of a Somigliana dislocation produced by a particular $\mathbf{b}(\mathbf{x})$ that smoothens out the step discontinuity, so to mitigate the stress singularities. This procedure has been implicitly considered in the perturbation of the edge dislocations presented in Section 2, where the Burger's vector $\mathbf{b}(\mathbf{x})$ was suitably tapered near the tip. Finding explicit conditions for the finiteness of strain has been the subject of various studies, starting from the 1902 work by Michele Gebbia, referred to in (Eshelby, 1951). Some analytic expressions to fillet the profile of a physical dislocation to produce an "equivalent" tapered Somigliana dislocation are reported in (Eshelby, 1951), Section 3.

For simplicity, we refer to the case in which the surface $\omega$ is planar. Introduce the reference system $\left(x_{1}, x_{2}, x_{3}\right)$, with corresponding unit vectors $\left(\mathbf{e}_{1}, \mathbf{e}_{2}, \mathbf{e}_{3}\right)$, such that $\omega$ is contained in the plane $\left(x_{1}, x_{2}\right)$ and indicate with $\omega^{+}$ and $\omega^{-}$the faces of $\omega$ at $x_{3}=0^{+}$and $x_{3}=0^{-}$, respectively. After the "cut and paste" of the new material, $\omega$ is separated in the two surfaces $\pi^{+}$and $\pi^{-}$, with $\omega^{+} \rightarrow \pi^{+}$and $\pi^{-} \rightarrow \omega^{-}$. Generically assume that $\mathbf{b}(\mathbf{x})=\phi\left(x_{1}, x_{2}\right) \mathbf{e}_{3}$, i.e., the separation occurs in the direction orthogonal to $\omega$, being $\phi\left(x_{1}, x_{2}\right)$ smooth enough to produce a sufficiently regular strain field inside the body. The surface of the extra-substance is identified by the mapping $\mathbf{y}^{ \pm}(\mathbf{x}): \omega \rightarrow \pi^{ \pm}$defined as

$$
\mathbf{y}^{ \pm}(\mathbf{x})=\mathbf{x}+\phi^{ \pm}(\mathbf{x}) \mathbf{e}_{3}, \mathbf{x} \in \omega
$$

with $\left[\phi^{+}(\mathbf{x})-\phi^{-}(\mathbf{x})\right] \mathbf{e}_{3}=\phi(\mathbf{x}) \mathbf{e}_{3}=\mathbf{b}(\mathbf{x}), \mathbf{x} \in \omega$. Let the state of stress that is generated in the body $\Omega$ after the pasting of the new material be represented by the first Piola Kirchhoff stress tensor $\mathbf{S}: \Omega \rightarrow$ Lin, associated with the deformation gradient $\mathbf{F}: \Omega \rightarrow \operatorname{Lin}^{+}$.

Consider now a closed pillow surface $\varpi$ that embraces the surface $\omega$, that in the limit will be made to shrink on $\omega$ so that, with intuitive notation, $\varpi^{+} \rightarrow \omega^{+}$and $\varpi^{-} \rightarrow \omega^{-}$. Let $\mathbf{N}$ generically denote the outward unit normal to $\varpi$, with $\mathbf{N}^{+}$and $\mathbf{N}^{-}$specifically orthogonal to $\varpi^{+}$and $\varpi^{-}$, respectively. We are interested in calculating the components of the configurational force parallel to $\omega$. For the component in the generic $\mathbf{e}_{1}$ direction, one has 


$$
\lim _{\varpi \rightarrow \omega} \mathbf{e}_{1} \cdot \int_{\omega} \mathbf{F}^{T} \mathbf{S N} d A=\int_{\omega^{+}} \mathbf{S} \mathbf{N}^{+} \cdot \mathbf{F} \mathbf{e}_{1} d A+\int_{\omega^{-}} \mathbf{S N}^{-} \cdot \mathbf{F} \mathbf{e}_{1} d A
$$

On $\varpi^{+} \rightarrow \omega^{+}$and $\varpi^{-} \rightarrow \omega^{-}$the quantity $\mathbf{F e}_{1}$ can be calculated from the only knowledge of the surface deformation field indicated by (4.1). Denoting with $S_{i j}=\mathbf{S} \cdot\left(\mathbf{e}_{i} \otimes \mathbf{e}_{j}\right)$, one finds that

$$
\begin{aligned}
\int_{\omega^{+}} \mathbf{S N}^{+} \cdot \mathbf{F} \mathbf{e}_{1} d A & +\int_{\omega^{-}} \mathbf{S N}^{-} \cdot \mathbf{F} \mathbf{e}_{1} d A=\int_{\omega^{+}} \mathbf{S} \mathbf{N}^{+} \cdot\left(\mathbf{e}_{1}+\phi_{, 1}^{+} \mathbf{e}_{3}\right) d A+\int_{\omega^{-}} \mathbf{S} \mathbf{N}^{-} \cdot\left(\mathbf{e}_{1}+\phi_{, 1}^{-} \mathbf{e}_{3}\right) d A \\
= & \mathbf{e}_{1} \cdot\left[\int_{\omega^{+}} \mathbf{S} \mathbf{N}^{+} d A+\int_{\omega^{-}} \mathbf{S N}^{-} d A\right]+\int_{\omega^{+}} \phi_{, 1}^{+} S_{33} d A-\int_{\omega^{+}} \phi_{, 1}^{-} S_{33} d A=\int_{\omega^{+}} \phi_{, 1}^{+} S_{33} d A-\int_{\omega^{+}} \phi_{, 1}^{-} S_{33} d A
\end{aligned}
$$

Here, we have used the fact that the sum of the integrals inside the square brackets is null because the inclusion must be in equilibrium and that $\mathbf{N}^{+}=-\mathbf{N}^{-}$when $\varpi^{+} \rightarrow \omega^{ \pm}$.

When the deformation is symmetric, so that $\phi^{+}(\mathbf{x})=-\phi^{-}(\mathbf{x})$, the limit value of the strain energy $\Psi$ is the same when approaching the same point on $\omega$ from the positive of negative direction of $\mathbf{e}_{3}$. Since $\mathbf{N}^{+}=-\mathbf{N}^{-}$one has that in the Eshelby intergral the contribution associated with $\Psi$ is null in the limit $\varpi \rightarrow \omega$. Moreover, clearly the limit of $S_{33}$ is the same on $\omega^{ \pm}$. Therefore, under the symmetry hypothesis, from (4.3) one has that

$$
\lim _{\varpi \rightarrow \omega} \mathbf{e}_{i} \cdot \int_{\varpi}\left(\Psi \mathbf{I}-\mathbf{F}^{T} \mathbf{S}\right) \mathbf{N} d A=\int_{\omega}-\phi_{, i} S_{33} d A, i=1,2,
$$

with $\phi=\phi^{+}-\phi^{-}$.

The Newtonian interpretation of such forces consists in assuming a particular type of contact between the matrix and the inclusion, at the interface $\pi^{+} \cup \pi^{-}$defined as above. Denote with $\mathbf{n}^{+}$and $\mathbf{n}^{+}$the outward unit normal to $\pi^{+}$and $\pi^{-}$, respectively, and with $d a$ the elementary portion of area on such surfaces. The element $d A$ with normal $\mathbf{N}^{ \pm}$on $\omega^{ \pm}$is then mapped to the element $d a$ with normal $\mathbf{n}^{ \pm}$on $\pi^{ \pm}$. A key point is to assume that the contact between the inclusion and the body matrix is frictionless. Moreover, at every point along the boundary, the component of the contact force in the direction of the vector $\mathbf{b}$ equals the component of the contact force predicted by the elastic solution for the Somigliana dislocation problem (where the adhesion between the inclusion and the remaining part of the material is supposed perfect). A physical interpretation of this assumption will be provided in Section 4.2, by using a boundary layer argument.

Let then $\mathbf{T}$ denote the Cauchy-stress tensor field associated with the first Piola-Kirchhoff stress $\mathbf{S}$. Under the aforementioned assumptions, observing that the direction of $\mathbf{b}$ is the same of $\mathbf{N}$ for the case at hand, the contact force acting on the elementary area $d a$ of $\pi^{ \pm}$is given by 


$$
d \mathbf{f}^{ \pm}=\frac{\mathbf{n}^{ \pm}}{\mathbf{n}^{ \pm} \cdot \mathbf{N}^{ \pm}}\left(\mathbf{N}^{ \pm} \cdot \mathbf{T n}^{ \pm}\right) d a
$$

Clearly, from the definition of stress representations, one has that $\mathbf{T n}^{ \pm} d a=\mathbf{S N}^{ \pm} d A$. Moreover, from (4.1),

$$
\mathbf{n}^{ \pm} d a= \pm\left(-\phi_{,, 1}^{ \pm} \mathbf{e}_{1}-\phi_{, 2}^{ \pm} \mathbf{e}_{2}+\mathbf{e}_{3}\right) d A, \mathbf{n}^{ \pm} \cdot \mathbf{N}^{ \pm}=\frac{d A}{d a} .
$$

Therefore

$$
\int_{\pi^{ \pm}} \frac{\mathbf{n}^{ \pm}}{\mathbf{n}^{ \pm} \cdot \mathbf{N}^{ \pm}}\left(\mathbf{N}^{ \pm} \cdot \mathbf{T n}^{ \pm}\right) d a=\int_{\omega^{ \pm}} \frac{ \pm\left(-\phi_{, 1}^{ \pm} \mathbf{e}_{1}-\phi_{, 2}^{ \pm} \mathbf{e}_{2}+\mathbf{e}_{3}\right) d A / d a}{d A / d a}\left(\mathbf{N}^{ \pm} \cdot \mathbf{S} \mathbf{N}^{ \pm}\right) d A=\int_{\omega^{ \pm}} \pm\left(-\phi_{, 1}^{+} \mathbf{e}_{1}-\phi_{, 2}^{+} \mathbf{e}_{2}+\mathbf{e}_{3}\right) S_{33} d A .
$$

Substituting in (4.5), recalling that $S_{33}$ is assumed to be continous on $\omega$, one finds that

$$
\int_{\pi^{+}} d \mathbf{f}^{+}+\int_{\pi^{-}} d \mathbf{f}^{-}=\int_{\omega^{+}}\left(-\phi_{, 1}^{+} \mathbf{e}_{1}-\phi_{, 2}^{+} \mathbf{e}_{2}+\mathbf{e}_{3}\right) S_{33} d A-\int_{\omega^{-}}\left(-\phi_{, 1}^{-} \mathbf{e}_{1}-\phi_{, 2}^{-} \mathbf{e}_{2}+\mathbf{e}_{3}\right) S_{33} d A=\int_{\omega}\left(-\phi_{,, 1} \mathbf{e}_{1}-\phi_{, 2} \mathbf{e}_{2}\right) S_{33} d A .
$$

This expression is identical to (4.4).

In the case in which the Somigliana dislocation presents a strongly singular stress field at the borders of $\omega$, associated with an irregular $\phi(\mathbf{x})$ like the stepped function of a physical dislocation, the derivation just obtained needs to be complemented by an additional argument.

Beside the elastic state associated with this configuration, referred to as state "state 0", consider a sequence of states characterized by the parameter $\lambda, \lambda \in[0,1]$, characterized through an expression analogous to (4.1) by the function $\phi_{\lambda}(\mathbf{x})=\phi_{\lambda}^{+}(\mathbf{x})-\phi_{\lambda}^{-}(\mathbf{x})$. We assume that $\phi_{\lambda}(\mathbf{x})=\phi_{0}(\mathbf{x})$ as $\lambda \rightarrow 0$ for $\mathbf{x} \in \omega$ (pointwise convergence), so that the sequence of surfaces representing the boundary of the inclusion forms a monotone non-intersecting homotopy. In particular, one should choose the sequence in such a way that, for any fixed $\lambda \in(0,1]$, the functions $\phi_{\lambda}^{+}(\mathbf{x})$ and $\phi_{\lambda}^{-}(\mathbf{x})$ are regular, and the corresponding strain energy $\Psi_{\lambda}(\mathbf{x})$ is integrable on $\omega$ (in the sense of the Cauchy principal value for the function $\left.\Psi_{\lambda}(\mathbf{x})\left(\mathbf{N}^{+}+\mathbf{N}^{-}\right), \mathbf{x} \in \omega\right)$. This procedure has been implicitly assumed for the perturbation of the tip of an edge dislocation, as represented in Figure 4 and Figure 5. The perturbation is intended to be "small", so that on a surface $\Theta$ that embraces $\omega$, sufficiently far from it, the stress and strain for the states $\lambda \in[0,1]$, coincide in practice. For the specific case of an edge dislocations, possible choices of $\phi_{\lambda}(\mathbf{x})$ that achieve this result are also indicated in (Eshelby, 1951), Section 3. Therefore 


$$
\int_{\Theta}\left(\Psi_{0} \mathbf{I}-\left(\mathbf{F}_{0}\right)^{T} \mathbf{S}_{0}\right) \mathbf{N} d A=\int_{\Theta}\left(\Psi_{\lambda} \mathbf{I}-\left(\mathbf{F}_{\lambda}\right)^{T} \mathbf{S}_{\lambda}\right) \mathbf{N} d A, \lambda \in[0,1]
$$

Due to the assumed regularity of $\phi_{\lambda}^{ \pm}(\mathbf{x}), \lambda \in(0,1]$, one can repeat the same argument used in this section to demonstrate that

$$
\lim _{\varpi \rightarrow \omega} \int_{\varpi}\left(\Psi_{\lambda} \mathbf{I}-\left(\mathbf{F}_{\lambda}\right)^{T} \mathbf{S}_{\lambda}\right) \mathbf{N} d A=\lim _{\omega \rightarrow \omega} \int_{\varpi}-\left(\mathbf{F}_{\lambda}\right)^{T} \mathbf{S}_{\lambda} \mathbf{N} d A=\int_{\omega}\left(-\phi_{\lambda, 1} \mathbf{e}_{1}-\phi_{\lambda, 2} \mathbf{e}_{2}\right) S_{\lambda 33} d A, \lambda \in(0,1],
$$

where $\mathbf{S}_{\lambda}=\mathbf{S}_{\lambda i j} \mathbf{e}_{i} \otimes \mathbf{e}_{j}$ refers to the stress in the configuration $\lambda$. Moreover, because of the path-independence of the flux of the Eshelby energy-momentum tensor and (4.9), one can write

$$
\begin{aligned}
\lim _{\varpi \rightarrow \omega} \int_{\varpi}\left(\Psi_{\lambda} \mathbf{I}-\left(\mathbf{F}_{\lambda}\right)^{T} \mathbf{S}_{\lambda}\right) \mathbf{N} d A=\int_{\Theta}\left(\Psi_{\lambda} \mathbf{I}-\left(\mathbf{F}_{\lambda}\right)^{T} \mathbf{S}_{\lambda}\right) \mathbf{N} d A \\
\quad=\int_{\Theta}\left(\Psi_{0} \mathbf{I}-\left(\mathbf{F}_{0}\right)^{T} \mathbf{S}_{0}\right) \mathbf{N} d A=\int_{\Pi}\left(\Psi_{0} \mathbf{I}-\left(\mathbf{F}_{0}\right)^{T} \mathbf{S}_{0}\right) \mathbf{N} d A, \lambda \in(0,1],
\end{aligned}
$$

where $\Pi$ is an arbitrary surface embracing $\omega$. Then, from (4.10) and (4.11), one obtains that

$$
\int_{\omega}\left(-\phi_{\lambda, 1} \mathbf{e}_{1}-\phi_{\lambda, 2} \mathbf{e}_{2}\right) S_{\lambda 33} d A=\int_{\Pi}\left(\Psi_{0} \mathbf{I}-\left(\mathbf{F}_{0}\right)^{T} \mathbf{S}_{0}\right) \mathbf{N} d A, \quad \lambda \in(0,1] .
$$

Then, taking the limit as $\lambda \rightarrow 0$, the conclusion is

$$
\lim _{\lambda \rightarrow 0} \int_{\omega}\left(-\phi_{\lambda, 1} \mathbf{e}_{1}-\phi_{\lambda, 2} \mathbf{e}_{2}\right) S_{\lambda 33} d A=\int_{\Pi}\left(\Psi_{0} \mathbf{I}-\left(\mathbf{F}_{0}\right)^{T} \mathbf{S}_{0}\right) \mathbf{N} d A .
$$

This limit should be considered as the convergence in the measure for the derivatives of $\phi_{\lambda}$ in the sense of distributions. This renders more rigorous the procedure used in Section 2 to regularize the step function of an edge dislocation. For such a case, the sequence of gradients of the perturbed profiles converges, in the aforementioned sense, to the Dirac's delta, the generalized derivate of the Heaviside function.

\subsection{By insight: a boundary layer theory}

The particular type of contact that has been assumed between the inclusion and the surrounding matrix may have, albeit tentatively, a definite physical interpretation that recalls, mutatis mutandis, the theory of the aerodynamic Boundary Layer (BL), first advanced by Ludwig Prandtl. This ingenuous approach, at the base of the engineering calculations of the effects of fluid flow around solids, allows substantial simplifications in the governing Navier-Stokes equations. The idea is to divide the flow field into two distinct areas: one inside 
the BL, dominated by viscosity, and the other outside the BL, where the effects of viscosity are negligible. The BL is characterized under the hypothesis that: i) the pressure distribution in the direction normal to it is constant; ii) the flow velocity is zero on the touched surface (no-slip condition); iii) its thickness is the distance from the solid surface at which the viscous flow velocity is almost equal to (it is customary to require $99 \%$ of) the freestream velocity, i.e., the velocity of an inviscid flow. Very simple elegant solutions can be obtained under the limit-case hypothesis that the BL thickness is much smaller than the characteristic size of the body invested by the fluid flow, so that it can be considered infinitesimal. In this case, the flow outside the BL can be determined by solving the problem of an inviscid fluid flow with Euler's equation of fluid dynamics, neglecting the thickness of the BL. Inside the BL, the viscous flow is found from the hypotheses of constant normal pressure, no slip condition and by assuming that, on the external surface, the fluid velocity is the solution of the aforementioned inviscid fluid problem.

No motion is allowed to an inclusion in equilibrium inside a solid body. However, most solids can be regarded as fluids with exceptionally high viscosity: permanent deformations can be obtained if a long enough time is allowed for creep effects to occur. Deformations of this type are more likely to start at the interface with the inclusion, where usually the stress is the highest. Varying the timescale, the long-term response of a solids may result to be comparable with the short-term response of a fluid. Similarly to the aerodynamic boundary layer theory, we can assume that, if a sufficient time is allowed, the matrix in which the inclusion is embedded can be distinguished into two distinct areas: a boundary layer wrapping the inclusion, where viscosity-induced deformations are dominant, and the remaining part of the body, where the effects of long term viscosity can be neglected and the response is typical of an elastic solids. In other words, inside the boundary layer the response of the matrix is similar to that of a fluid. Because of this, a certain degree of freedom is allowed to the inclusion, which tends to move because subjected to a non-zero force resultant.

The contact forces exerted at the interface with the inclusion are governed by the assumed presence of the boundary layer, supposed on infinitesimal thickness. The underlying hypothesis for their calculation is that the contact with the inclusion at the inner surface of the BL is frictionless. This is the perfect-slip condition, which represents the counterpart of the no-slip condition for the aerodynamic BL. On the external surface of the BL the state of stress is that resulting from the elastic problem in which, neglecting the thickness of the BL itself, the inclusion is supposed to be perfectly adherent with the elastic matrix. The further condition to be included is that the component of the contact forces at the interface with the inclusion in the direction of the displacement vector b remains constant throughout the boundary layer. In the aerodynamic BL analogy, this is the counterpart of the constancy of the normal pressure distribution. For the case of inclusions, the validity of the constant-in-the-thickness hypothesis is justified by the fact that the boundary layer is assumed to be extremely thin. Then, such a condition can be derived from a criterion of energetic equivalence, in terms of the work that is necessary to insert the inclusion inside the matrix. In fact, the relative displacement of the resulting surfaces after the cut of $\omega$ occurs in the direction of the vector $\mathbf{b}$, whereas the forces dual in energy to such a 
displacement are indeed the components of the contact forces in the direction of $\mathbf{b}$ itself. In the limit of evanescent thickness of the BL, this is equivalent to state that no energy is associated with its deformation.

\section{CONCLUSIONS}

We have proposed an asymptotic perturbation approach to calculate the configurational forces acting on dislocations and cracks. The classical balance laws for the Newtonian contact forces, acting on the perturbed configuration of an inclusion equivalent to an edge dislocation, directly furnish an unbalanced resultant provided that a particular frictionless contact is assumed between the matrix and the equivalent inclusion. This force coincides, in the limit in which the original shape is restored, with the well-known configurational force obtained via energy-based arguments. For cracks, the perturbation consists in considering the cohesive model à la Barenblatt, recovering the configuration of a sharp crack in the small-scale bridging limit. Equilibrium in the deformed state of the bundle of cohesive ligaments forming the process zone, here intended as an object of substance called the cohesive block, requires the balance between the driving force, induced by the frictionless sliding of the cohesive block at the interface, and the restoring force, that opposes such sliding. For sharp cracks, Griffith energetic balance is thus interpreted as the balance of the linear momentum for the material point representing the small-scale-bridging limit of the cohesive block, located at the crack tip.

This procedure has been generalized in more rigorous terms for the paradigmatic case of a Somigliana dislocation, providing as well an intuitive interpretation for the special nature of the contact between the equivalent inclusion and surrounding matrix via the presence of a thin boundary layer. Although further speculations will certainly be required, we believe that the proposed Newtonian interpretation of the configurational forces on defects is alternative and complementary to the classical energy-based derivation, according to which they are defined by the energy release rate of the moving defect. The cases presented in this article refer to the examples of edge or Somigliana dislocations and straight cracks. The cases of shear forces acting on dislocations, or the opening of cracks in mode II or mode III, still need to be determined. The extension to three-dimensional problems such as penny-shape cracks, also in presence of material inhomogeneity, presents no conceptual difficulty. The same formalism could also be applied on timedependent situations, by introducing time as an extra independent variable, and accounting for inertia forces in the linear and angular momentum balance.

We believe that the interpretation of the configurational forces presented here is physically intuitive and might help to increase the confidence in these objects, by permitting their geometric visualization and promoting their use with the classical "taste" of Newtonian mechanics. 


\section{ACKNOWLEDGEMENT}

R.B. acknowledges the Thomas and Laura Hsu Professorship and the National Science Foundation under Grant CMMI -1361868. G.R.C. acknowledges the support of the European Community under Contract RFSR-CT2012-00026 (S+G RFS-PR-11017). This work was performed while G.R.C. was a visiting Professor at the University of Houston, TX.

\section{REFERENCES}

Balabukh, L., Vulfson, M., Mukoseev, B. \& Panovko, Y., 1970. On work done by reaction forces on moving supports. Research on Theory of Construction (in Russian), Volume 18, pp. 190-200.

Barenblatt, G. I., 1962. The mathematical theory of equilibrium cracks in brittle fracture. Advances in Applied Mechanics, 7(C), pp. 55-129.

Bigoni, D., Bosi, F., Dal Corso, F. \& Misseroni, D., 2014. Instability of a penetrating blade. Journal of the Mechanics and Physics of Solids, Volume 2014, pp. 411-425.

Bigoni, D., Dal Corso, F., Bosi, F. \& Missoroni, D., 2015. Eshelby-like forces acting on elastic structures: Theoretical and experimental proof. Mechanics of Materials, Volume 80, pp. 368-374.

Bigoni, D., Dal Corso, F., Misseroni, D. \& Bosi, F., 2014. Torsional locomotion. Proceedings of the Royal Society A: Mathematical, Physical and Engineering Sciences, Volume 470, p. 20140599.

Bosi, F., Misseroni, D., Dal Corso, F. \& Bigoni, D., 2014. An elastica arm scale. Preceedings of the Royal Society A: Mathematical, Physical and Engineering Sciences, Volume 470, p. 20140232.

Bosi, F., Misseroni, D., Dal Corso, F. \& Bigoni, D., 2015. Development of configurational forces during the injection of an elastic rod. Extreme Mechanics Letters, Volume 4, pp. 83-88.

Bosi, F., Misseroni, D., Dal Corso, F. \& Bigoni, D., 2015. Self-encapsulation, or the "dripping" of an elastic rod. Proceedings of the Royal Society A, 471(2179), p. 20150195.

Dundurs, J., 1969. Elastic Interaction of dislocations with inhomogeneities. In: T. Mura, ed. Mathematical Theory of Dislocations. New York: ASME, pp. 70-115.

Eshelby, J., 1975. The elastic energy-momentum tensor. Journal of Elasticity, 5(3-4), pp. 321-335.

Eshelby, J. D., 1951. The force on elastic singularity. Philosophical Transaction of the Royal Society of London, Series A, Mathematical and Physical Sciences, Volume 877, pp. 87-112.

Gurtin, M., 1995. The nature of configurational forces. Archive for Rational Mechanics and Analysis, Volume 131, pp. 67-100.

Gurtin, M., 2008. Configurational Forces as Basic Concepts of Continuum Physics. New York: Springer Science \& Business Media.

Gurtin, M. \& Podio-Guidugli, P., 1996. Configurational forces and the basic laws of crack propagation. Journal of the Mechanics and Physics of Solids, Volume 44, pp. 905-927. 
Rice, J. R., 1968. A path independent integral and the approximate analysis of strain concentration by notches and cracks. Journal of Applied Mechanics, 35(2), pp. 379-386.

Truesdell, C., 1966. Method and taste in natural phylosophy. In: Six Lectures on Modern Natural Philosophy. New York: Springer Verlag.

Volterra, V., 1907. Sur l'équilibre des corps élastiques multiplement connexes. Annales Scientifiques de École Normale Supérieure, Sér 3, Volume 24, pp. 401-517.

Willis, J., 1967. A comparison of the fracture criteria of Griffith and Barenblatt. Journal of the Mechanics and Physics of Solids, Volume 15, pp. 151-162. 